\title{
FULL DISCRETIZATION OF SEMILINEAR STOCHASTIC WAVE EQUATIONS DRIVEN BY MULTIPLICATIVE NOISE*
}

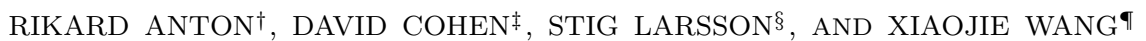

\begin{abstract}
A fully discrete approximation of the semilinear stochastic wave equation driven by multiplicative noise is presented. A standard linear finite element approximation is used in space, and a stochastic trigonometric method is used for the temporal approximation. This explicit time integrator allows for mean-square error bounds independent of the space discretization and thus does not suffer from a step size restriction as in the often used Störmer-Verlet leapfrog scheme. Furthermore, it satisfies an almost trace formula (i.e., a linear drift of the expected value of the energy of the problem). Numerical experiments are presented and confirm the theoretical results.
\end{abstract}

Key words. semilinear stochastic wave equation, multiplicative noise, strong convergence, trace formula, stochastic trigonometric methods, geometric numerical integration

AMS subject classifications. 65C20,60H10, 60H15, 60H35, 65C30

DOI. $10.1137 / 15 \mathrm{M} 101049 \mathrm{X}$

1. Introduction. We consider the numerical discretization of semilinear stochastic wave equations of the form

$$
\begin{array}{ll}
\mathrm{d} \dot{u}-\Delta u \mathrm{~d} t=f(u) \mathrm{d} t+g(u) \mathrm{d} W & \text { in } \mathcal{D} \times(0, \infty), \\
u=0 & \text { in } \partial \mathcal{D} \times(0, \infty), \\
u(\cdot, 0)=u_{0}, \dot{u}(\cdot, 0)=v_{0} & \text { in } \mathcal{D},
\end{array}
$$

where $u=u(x, t)$ and $\mathcal{D} \subset \mathbb{R}^{d}, d=1,2,3$, is a bounded convex domain with polygonal boundary $\partial \mathcal{D}$. The "." denotes the time derivative $\frac{\partial}{\partial t}$. Assumptions on the smoothness of the nonlinearities $f$ and $g$ will be given below. The stochastic process $\{W(t)\}_{t \geq 0}$ is an $L_{2}(\mathcal{D})$-valued (possibly cylindrical) $Q$-Wiener process with respect to a normal filtration $\left\{\mathcal{F}_{t}\right\}_{t \geq 0}$ on a filtered probability space $\left(\Omega, \mathcal{F}, \mathbb{P},\left\{\mathcal{F}_{t}\right\}_{t \geq 0}\right)$. The initial data $u_{0}$ and $v_{0}$ are $\mathcal{F}_{0}$-measurable random variables. We will numerically solve this problem with a linear finite element method in space and a stochastic trigonometric method in time.

We refer the reader to the introductions of [16] and [5] for relevant literature on the spatial, respectively, temporal, discretization of stochastic (linear) wave equations. Further, the recent publication [22] presents a full discretization of the wave equation with additive noise: a spectral Galerkin approximation is used in space, and an

${ }^{*}$ Received by the editors February 27, 2015; accepted for publication (in revised form) February 3, 2016; published electronically April 12, 2016. This work was partially supported by UMIT Research Lab at Umeå University, the Swedish Research Council (VR) (project 2013-4562), and the National Natural Science Foundations of China (project 11301550). The computations were performed on resources provided by the Swedish National Infrastructure for Computing (SNIC) at HPC2N, Umeå University.

http://www.siam.org/journals/sinum/54-2/M101049.html

†Department of Mathematics and Mathematical Statistics, Umeå University, SE-901 87 Umeå, Sweden (rikard.anton@umu.se).

‡Department of Mathematics and Mathematical Statistics, Umeå University, SE-901 87 Umeå, Sweden, and Department of Mathematics, University of Innsbruck, A-6020 Innsbruck, Austria (david.cohen@umu.se, david.cohen@uibk.ac.at).

$\S$ Department of Mathematical Sciences, Chalmers University of Technology and University of Gothenburg, SE-41296 Gothenburg, Sweden (stig@chalmers.se).

ISchool of Mathematics and Statistics, Central South University, CN-410083 Changsha, Hunan, China (x.j.wang7@csu.edu.cn). 
adapted stochastic trigonometric method, using linear functionals of the noise as in [12], is employed in time. Furthermore, the time discretization of nonlinear stochastic wave equations by stochastic trigonometric methods is analyzed in [21]. Finally, let us mention the recent publication [6], which analyzes convergence in $L^{p}(\Omega)$ of the stochastic trigonometric method applied to the one-dimensional nonlinear stochastic wave equation.

In the present paper, we prove mean-square convergence for the full discretization to the exact solution to the nonlinear problem (1). Furthermore, using this result, we derive a geometric property of our numerical integrator, namely, a trace formula. The trace formula (the linear drift of the expected value of the energy) for the exact solution of (1), as well as for the finite element solution and the completely discrete solution, is presented.

Strong approximations of stochastic wave equations are relevant in many real applications. For example, let us consider the motion of a strand of DNA floating in a liquid, as presented in [9] and references therein. The motion of the DNA molecule may be modeled by a wave equation, and the impact of the fluid's molecules may be modeled by a stochastic force acting on the string. When two normally distant parts of the DNA get close enough, biological events, such as release of enzymes, occur. It is thus of interest to consider strong approximation of stochastic wave equations in such a situation.

The paper is organized as follows. We introduce some notation and mention some useful results in section 2. Section 3 presents a mean-square convergence analysis for our numerical discretization. A trace formula for the exact and numerical solutions is given in section 4. Numerical experiments illustrating the rates of convergence and the trace formula of the numerical solution are given in section 5. Finally, an appendix is given in section 6 .

2. Notation and useful results. Let $U$ and $H$ be separable Hilbert spaces with norms $\|\cdot\|_{U}$ and $\|\cdot\|_{H}$, respectively. We denote the space of bounded linear operators from $U$ to $H$ by $\mathcal{L}(U, H)$, and we let $\mathcal{L}_{2}(U, H)$ be the set of Hilbert-Schmidt operators with norm

$$
\|T\|_{\mathcal{L}_{2}(U, H)}:=\left(\sum_{k=1}^{\infty}\left\|T e_{k}\right\|_{H}^{2}\right)^{1 / 2},
$$

where $\left\{e_{k}\right\}_{k=1}^{\infty}$ is an arbitrary orthonormal basis of $U$. If $H=U$, then we write $\mathcal{L}(U)=$ $\mathcal{L}(U, U)$ and $\mathrm{HS}=\mathcal{L}_{2}(U, U)$. Let $Q \in \mathcal{L}(U)$ be a self-adjoint, positive semidefinite operator. We denote the space of Hilbert-Schmidt operators from $Q^{1 / 2}(U)$ to $H=U$ by $\mathcal{L}_{2}^{0}$ with norm

$$
\|T\|_{\mathcal{L}_{2}^{0}}=\left\|T Q^{1 / 2}\right\|_{\mathrm{HS}}
$$

For the stochastic wave equation $(1)$, we define $U:=L_{2}(\mathcal{D})$ and denote the $L_{2}(\mathcal{D})$ norm by $\|\cdot\|:=\|\cdot\|_{L_{2}(\mathcal{D})}$. Further, we set $\Lambda=-\Delta$ with $D(\Lambda)=H^{2}(\mathcal{D}) \cap H_{0}^{1}(\mathcal{D})$.

Let $\left(\Omega, \mathcal{F}, \mathbb{P},\left\{\mathcal{F}_{t}\right\}_{t \geq 0}\right)$ be a filtered probability space, and let $L_{2}(\Omega, H)$ be the space of $H$-valued square integrable random variables with norm

$$
\|v\|_{L_{2}(\Omega, H)}:=\mathbb{E}\left[\|v\|_{H}^{2}\right]^{1 / 2} .
$$

Next, we define the space $\dot{H}^{\alpha}=D\left(\Lambda^{\alpha / 2}\right)$, for $\alpha \in \mathbb{R}$, with norm

$$
\|v\|_{\alpha}:=\left\|\Lambda^{\alpha / 2} v\right\|_{L_{2}(\mathcal{D})}=\left(\sum_{j=1}^{\infty} \lambda_{j}^{\alpha}\left(v, \varphi_{j}\right)_{L_{2}(\mathcal{D})}^{2}\right)^{1 / 2},
$$

Copyright $@$ ( ) by SIAM. Unauthorized reproduction of this article is prohibited. 
where $\left\{\left(\lambda_{j}, \varphi_{j}\right)\right\}_{j=1}^{\infty}$ are the eigenpairs of $\Lambda$ with orthonormal eigenvectors. We also introduce the space

$$
H^{\alpha}:=\dot{H}^{\alpha} \times \dot{H}^{\alpha-1},
$$

with norm $\|v\|\left\|_{\alpha}^{2}:=\right\| v_{1}\left\|_{\alpha}^{2}+\right\| v_{2} \|_{\alpha-1}^{2}$, for $\alpha \in \mathbb{R}$ and $v=\left[v_{1}, v_{2}\right]^{T}$. Note that $\dot{H}^{0}=U:=L_{2}(\mathcal{D})$ and $H:=H^{0}=\dot{H}^{0} \times \dot{H}^{-1}$. In the following we denote the scalar product by $(\cdot, \cdot)=(\cdot, \cdot)_{L_{2}(\mathcal{D})}$ and recall the notation for the norm $\|\cdot\|=\|\cdot\|_{L_{2}(\mathcal{D})}$.

Denoting the velocity of the solution to our stochastic partial differential equation by $u_{2}:=\dot{u}_{1}:=\dot{u}$, one can rewrite (1) as

$$
\begin{aligned}
& \mathrm{d} X(t)=A X(t) \mathrm{d} t+F(X(t)) \mathrm{d} t+G(X(t)) \mathrm{d} W(t), \quad t>0, \\
& X(0)=X_{0},
\end{aligned}
$$

where

$X:=\left[\begin{array}{l}u_{1} \\ u_{2}\end{array}\right], A:=\left[\begin{array}{cc}0 & I \\ -\Lambda & 0\end{array}\right], F(X):=\left[\begin{array}{c}0 \\ f\left(u_{1}\right)\end{array}\right], G(X):=\left[\begin{array}{c}0 \\ g\left(u_{1}\right)\end{array}\right]$, and $X_{0}:=\left[\begin{array}{l}u_{0} \\ v_{0}\end{array}\right]$.

The operator $A$ with $D(A)=H^{1}=\dot{H}^{1} \times \dot{H}^{0}$ is the generator of a strongly continuous semigroup of bounded linear operators $E(t)=\mathrm{e}^{t A}$ on $H=H^{0}=\dot{H}^{0} \times \dot{H}^{-1}$ —in fact, a unitary group.

Let $\left\{\mathcal{T}_{h}\right\}$ be a quasi-uniform family of triangulations of the convex polygonal domain $\mathcal{D}$ with $h_{\mathrm{K}}=\operatorname{diam}(K)$ and $h=\max _{\mathrm{K} \in \mathcal{T}_{h}} h_{\mathrm{K}}$. Let $V_{h} \subset H_{0}^{1}(\mathcal{D})=\dot{H}^{1}$ be the space of piecewise linear continuous functions with respect to $\mathcal{T}_{h}$ which are zero on the boundary of $\mathcal{D}$, and let $\mathcal{P}_{h}: \dot{H}^{0} \rightarrow V_{h}$ denote the $\dot{H}^{0}$-orthogonal projector and $\mathcal{R}_{h}: \dot{H}^{1} \rightarrow V_{h}$ denote the $\dot{H}^{1}$-orthogonal projector (Ritz projector). Thus,

$$
\left(\mathcal{P}_{h} v, w_{h}\right)=\left(v, w_{h}\right), \quad\left(\nabla \mathcal{R}_{h} u, \nabla w_{h}\right)=\left(\nabla u, \nabla w_{h}\right) \quad \forall v \in \dot{H}^{0}, u \in \dot{H}^{1}, w_{h} \in V_{h} .
$$

The discrete Laplace operator $\Lambda_{h}: V_{h} \rightarrow V_{h}$ is then defined by

$$
\left(\Lambda_{h} v_{h}, w_{h}\right)=\left(\nabla v_{h}, \nabla w_{h}\right) \quad \forall w_{h} \in V_{h} .
$$

We note that $\mathcal{R}_{h}=\Lambda_{h}^{-1} \mathcal{P}_{h} \Lambda$. We also define discrete variants of $\|\cdot\|_{\alpha}$ and $\dot{H}^{\alpha}$ by

$$
\left\|v_{h}\right\|_{h, \alpha}=\left\|\Lambda_{h}^{\alpha / 2} v_{h}\right\|, \quad v_{h} \in V_{h},
$$

and $\dot{H}_{h}^{\alpha}=V_{h}$ equipped with the norm $\|\cdot\|_{h, \alpha}$. Finally, the finite element approximation of (1) can then be written as

$$
\begin{aligned}
& \mathrm{d} \dot{u}_{h, 1}(t)+\Lambda_{h} u_{h, 1}(t) \mathrm{d} t=\mathcal{P}_{h} f\left(u_{h, 1}(t)\right) \mathrm{d} t+\mathcal{P}_{h} g\left(u_{h, 1}(t)\right) \mathrm{d} W(t), \quad t>0, \\
& u_{h, 1}(0)=u_{h, 0}, u_{h, 2}(0)=v_{h, 0}
\end{aligned}
$$

or, in abstract form,

$$
\begin{aligned}
& \mathrm{d} X_{h}(t)=A_{h} X_{h}(t) \mathrm{d} t+\mathcal{P}_{h} F\left(X_{h}(t)\right) \mathrm{d} t+\mathcal{P}_{h} G\left(X_{h}(t)\right) \mathrm{d} W(t), \quad t>0, \\
& X_{h}(0)=X_{h, 0},
\end{aligned}
$$

where

$$
A_{h}:=\left[\begin{array}{cc}
0 & I \\
-\Lambda_{h} & 0
\end{array}\right], \quad X_{h}:=\left[\begin{array}{l}
u_{h, 1} \\
u_{h, 2}
\end{array}\right]
$$

Copyright $@$ by SIAM. Unauthorized reproduction of this article is prohibited. 
$F$ and $G$ are as before, and

$$
X_{h, 0}:=\left[\begin{array}{l}
u_{h, 0} \\
v_{h, 0}
\end{array}\right] \quad \text { with } u_{h, 0}=\mathcal{R}_{h} u_{0}, v_{h, 0}=\mathcal{P}_{h} v_{0} \in V_{h} .
$$

Note the abuse of notation for the projection $\mathcal{P}_{h} F\left(X_{h}\right)=\left(0, \mathcal{P}_{h} f\left(u_{h, 1}\right)\right)^{T}$ and similarly for $\mathcal{P}_{h} G\left(X_{h}\right)$. This will be used throughout the paper. Again, $A_{h}$ is the generator of a $C_{0}$-semigroup $E_{h}(t)=\mathrm{e}^{t A_{h}}$ on $H_{h}:=\dot{H}_{h}^{0} \times \dot{H}_{h}^{-1}$.

We study (2) and (4) in their mild forms

$X_{h}(t)=E_{h}(t) X_{h, 0}+\int_{0}^{t} E_{h}(t-s) \mathcal{P}_{h} F\left(X_{h}(s)\right) \mathrm{d} s+\int_{0}^{t} E_{h}(t-s) \mathcal{P}_{h} G\left(X_{h}(s)\right) \mathrm{d} W(s)$,

where the semigroups can be expressed as

$$
\begin{aligned}
E(t) & =\left[\begin{array}{cc}
C(t) & \Lambda^{-1 / 2} S(t) \\
-\Lambda^{1 / 2} S(t) & C(t)
\end{array}\right], \\
E_{h}(t) & =\left[\begin{array}{cc}
C_{h}(t) & \Lambda_{h}^{-1 / 2} S_{h}(t) \\
-\Lambda_{h}^{1 / 2} S_{h}(t) & C_{h}(t)
\end{array}\right],
\end{aligned}
$$

with $C(t)=\cos \left(t \Lambda^{1 / 2}\right), S(t)=\sin \left(t \Lambda^{1 / 2}\right), C_{h}(t)=\cos \left(t \Lambda_{h}^{1 / 2}\right)$, and $S_{h}(t)=\sin \left(t \Lambda_{h}^{1 / 2}\right)$.

In order to ensure existence and uniqueness of problem (1), we shall assume that $u_{0} \in L_{2}\left(\Omega, \dot{H}^{\gamma}\right)$ and $v_{0} \in L_{2}\left(\Omega, \dot{H}^{\gamma-1}\right)$, with $\gamma=\max (\beta, 1)$ for some regularity parameter $\beta \geq 0$, and that the functions $f: L_{2}(\mathcal{D}) \rightarrow L_{2}(\mathcal{D})$ and $g: L_{2}(\mathcal{D}) \rightarrow \mathcal{L}_{2}^{0}$ satisfy

$$
\begin{aligned}
\|f(u)-f(v)\|+\|g(u)-g(v)\|_{\mathcal{L}_{2}^{0}} \leq C\|u-v\| & & \text { if } \beta \geq 0, \\
\|f(u)\|+\left\|\Lambda^{(\beta-1) / 2} g(u)\right\|_{\mathcal{L}_{2}^{0}} \leq C(1+\|u\|) & & \text { if } 0 \leq \beta \leq 1, \\
\left\|\Lambda^{(\beta-1) / 2} f(u)\right\|+\left\|\Lambda^{(\beta-1) / 2} g(u)\right\|_{\mathcal{L}_{2}^{0}} \leq C\left(1+\left\|\Lambda^{(\beta-1) / 2} u\right\|\right) & & \text { if } \beta>1
\end{aligned}
$$

for all $u, v \in L_{2}(\mathcal{D})$ in the first two inequalities and for all $u \in \dot{H}^{\beta-1}$ in the last one. Throughout the text, $C$ (or $C_{1}, C_{2}, K_{1}, K_{2}$, etc.) denotes a generic positive constant that may vary from line to line. We assume that the order of initial regularity $\gamma \geq 1$ so that the discrete initial value $u_{h, 0}=\mathcal{R}_{h} u_{0}$ is well defined.

Lemma 1. Assume that $u_{0} \in L_{2}\left(\Omega, \dot{H}^{\gamma}\right), v_{0} \in L_{2}\left(\Omega, \dot{H}^{\gamma-1}\right)$ with $\gamma=\max (\beta, 1)$ and that the functions $f$ and $g$ satisfy (9) for some $\beta \geq 0$. Then there exists a unique solution to the stochastic wave equation (2) and the finite element equation (4) given by the solution of their respective mild equations, i.e., (5) and (7).

The proof of this lemma follows from [8, Theorem 7.4]; see also the proof of Theorem 2.1 in [21].

We now collect some results that we will use later. Sketches of the proofs of these results are collected in the appendix.

- The error estimates for the cosine and sine operators (Corollary 4.2 in [16]): Denote $X_{0}=\left[u_{0}, v_{0}\right]^{T}$ and let

$$
\begin{aligned}
& \mathcal{G}_{h}(t) X_{0}=\left(C_{h}(t) \mathcal{R}_{h}-C(t)\right) u_{0}+\left(\Lambda_{h}^{-1 / 2} S_{h}(t) \mathcal{P}_{h}-\Lambda^{-1 / 2} S(t)\right) v_{0}, \\
& \dot{\mathcal{G}}_{h}(t) X_{0}=-\left(\Lambda_{h}^{1 / 2} S_{h}(t) \mathcal{R}_{h}-\Lambda^{1 / 2} S(t)\right) u_{0}+\left(C_{h}(t) \mathcal{P}_{h}-C(t)\right) v_{0} .
\end{aligned}
$$

Copyright $\odot$ by SIAM. Unauthorized reproduction of this article is prohibited. 
Then we have

$$
\begin{aligned}
& \left\|\mathcal{G}_{h}(t) X_{0}\right\| \leq\left. C \cdot(1+t) \cdot h^{\gamma-1}\left\|X_{0}\right\|\right|_{\gamma}, \quad t \geq 0, \quad \gamma \in[1,3], \\
& \left\|\dot{\mathcal{G}}_{h}(t) X_{0}\right\| \leq C \cdot(1+t) \cdot h^{\frac{2}{3}(\gamma-1)}\left|\left\|X_{0} \mid\right\|_{\gamma}, \quad t \geq 0, \quad \gamma \in[1,4] .\right.
\end{aligned}
$$

These will be used to estimate the error contributions from the initial values. In order to deal with the convolution terms in (7), we single out the following error estimates. Let

$$
\begin{aligned}
& \mathcal{K}_{h}(t) v_{0}=\left(\Lambda_{h}^{-1 / 2} S_{h}(t) \mathcal{P}_{h}-\Lambda^{-1 / 2} S(t)\right) v_{0}, \\
& \dot{\mathcal{K}}_{h}(t) v_{0}=\left(C_{h}(t) \mathcal{P}_{h}-C(t)\right) v_{0} .
\end{aligned}
$$

Then we have

$$
\begin{aligned}
& \left\|\mathcal{K}_{h}(t) v_{0}\right\| \leq C \cdot(1+t) \cdot h^{\frac{2}{3} \beta}\left\|v_{0}\right\|_{\beta-1}, \quad t \geq 0, \quad \beta \in[0,3], \\
& \left\|\dot{\mathcal{K}}_{h}(t) v_{0}\right\| \leq C \cdot(1+t) \cdot h^{\frac{2}{3}(\beta-1)}\left\|v_{0}\right\|_{\beta-1}, \quad t \geq 0, \quad \beta \in[1,4] .
\end{aligned}
$$

- The temporal Hölder continuity of the sine and cosine operators (see (4.1) in $[5]):$

$$
\begin{aligned}
\left\|\left(S_{h}(t)-S_{h}(s)\right) \Lambda_{h}^{-\beta / 2}\right\|_{\mathcal{L}(U)} & \leq C \cdot|t-s|^{\beta}, & & \beta \in[0,1], \\
\left\|\left(C_{h}(t)-C_{h}(s)\right) \Lambda_{h}^{-(\beta-1) / 2}\right\|_{\mathcal{L}(U)} & \leq C \cdot|t-s|^{\beta-1}, & & \beta \in[1,2],
\end{aligned}
$$

together with its continuous version,

$$
\begin{aligned}
\left\|(S(t)-S(s)) \Lambda^{-\beta / 2}\right\|_{\mathcal{L}(U)} & \leq C \cdot|t-s|^{\beta}, & & \beta \in[0,1], \\
\left\|(C(t)-C(s)) \Lambda^{-(\beta-1) / 2}\right\|_{\mathcal{L}(U)} & \leq C \cdot|t-s|^{\beta-1}, & & \beta \in[1,2] .
\end{aligned}
$$

- The equivalence of $\Lambda_{h}$ and $\Lambda$ (see the proof of Theorem 4.4 in [15]): This uses an inverse inequality, hence our assumption about the quasi-uniformity of the mesh family:

$$
\left\|\Lambda_{h}^{\alpha} \mathcal{P}_{h} \Lambda^{-\alpha} v\right\|^{2} \leq\|v\|^{2}, \quad \alpha \in\left[-\frac{1}{2}, 1\right], \quad v \in \dot{H}^{0}=L_{2}(\mathcal{D}) .
$$

- The equivalence of the discrete and continuous norms (see (2.13) in [1]):

$$
c\left\|\Lambda_{h}^{\gamma} v_{h}\right\| \leq\left\|\Lambda^{\gamma} v_{h}\right\| \leq C\left\|\Lambda_{h}^{\gamma} v_{h}\right\| \quad \text { for } \quad v_{h} \in V_{h} \quad \text { and } \quad \gamma \in\left[-\frac{1}{2}, \frac{1}{2}\right] .
$$

Using the above estimates, one can deduce the following regularity results for the exact solution to our stochastic wave equation (1) and for the exact solution of the finite element approximation (3).

Proposition 2. Let $\left[u_{1}, u_{2}\right]^{T}$ be the solution to (1), where the initial values satisfy $u_{0} \in L_{2}\left(\Omega, \dot{H}^{\gamma}\right), v_{0} \in L_{2}\left(\Omega, \dot{H}^{\gamma-1}\right)$ with $\gamma=\max (\beta, 1)$, and the functions $f$ and $g$ satisfy (9) for some $\beta \geq 0$. Then it holds that

$$
\sup _{0 \leq t \leq T} \mathbb{E}\left[\left\|u_{1}(t)\right\|_{\beta}^{2}+\left\|u_{2}(t)\right\|_{\beta-1}^{2}\right] \leq C
$$

and, for $0 \leq s \leq t \leq T$,

$$
\begin{aligned}
\mathbb{E}\left[\left\|u_{1}(t)-u_{1}(s)\right\|^{2}\right] \leq C|t-s|^{2 \min (\beta, 1)}( & \mathbb{E}\left[\left\|u_{0}\right\|_{\beta}^{2}+\left\|v_{0}\right\|_{\beta-1}^{2}\right] \\
& \left.+\sup _{r \in[0, T]} \mathbb{E}\left[1+\left\|u_{1}(r)\right\|_{\beta}^{2}\right]\right) .
\end{aligned}
$$

Copyright (c) by SIAM. Unauthorized reproduction of this article is prohibited. 
The proof of this proposition is very similar to the proof of Proposition 3 given below and is therefore omitted (see also the proofs of Proposition 3.1 and Lemma 3.3 in [21]).

The next result will be useful in section 4 when we will deal with the trace formula of the numerical solution.

Proposition 3. Let $\left[u_{h, 1}, u_{h, 2}\right]^{T}$ be the solution to the finite element problem (3), where the initial values satisfy $u_{0} \in L_{2}\left(\Omega, \dot{H}^{\gamma}\right), v_{0} \in L_{2}\left(\Omega, \dot{H}^{\gamma-1}\right)$ with $\gamma=\max (\beta, 1)$, and the functions $f$ and $g$ satisfy (9) for some $\beta \in[0,2]$. Then it holds that

$$
\sup _{0 \leq t \leq T} \mathbb{E}\left[\left\|u_{h, 1}(t)\right\|_{h, \beta}^{2}+\left\|u_{h, 2}(t)\right\|_{h, \beta-1}^{2}\right] \leq C
$$

and, for $0 \leq s \leq t \leq T$,

$$
\begin{aligned}
\mathbb{E}\left[\left\|u_{h, 1}(t)-u_{h, 1}(s)\right\|^{2}\right] \leq C|t-s|^{2 \min (\beta, 1)}( & \mathbb{E}\left[\left\|u_{h, 0}\right\|_{h, \beta}^{2}+\left\|v_{h, 0}\right\|_{h, \beta-1}^{2}\right] \\
& \left.+\sup _{r \in[0, T]} \mathbb{E}\left[1+\left\|u_{h, 1}(r)\right\|_{h, \beta}^{2}\right]\right),
\end{aligned}
$$

where we recall that $u_{h, 0}$ and $v_{h, 0}$ are the initial position and velocity to the finite element problem.

Proof. Let us start with the first estimate of the norm of $\Lambda_{h}^{\beta / 2} u_{h, 1}(t)$ and consider the expression

$$
\begin{aligned}
\Lambda_{h}^{\beta / 2} u_{h, 1}(t)= & \Lambda_{h}^{\beta / 2} C_{h}(t) u_{h, 0}+\Lambda_{h}^{(\beta-1) / 2} S_{h}(t) v_{h, 0} \\
& +\int_{0}^{t} \Lambda_{h}^{(\beta-1) / 2} S_{h}(t-r) \mathcal{P}_{h} f\left(u_{h, 1}(r)\right) \mathrm{d} r \\
& +\int_{0}^{t} \Lambda_{h}^{(\beta-1) / 2} S_{h}(t-r) \mathcal{P}_{h} g\left(u_{h, 1}(r)\right) \mathrm{d} W(r) .
\end{aligned}
$$

Using the fact that $\Lambda_{h}$ and $C_{h}(t)$ commute and the boundedness of the cosine operator together with our assumptions on the initial values for the finite element problem, we get

$$
\mathbb{E}\left[\left\|\Lambda_{h}^{\beta / 2} C_{h}(t) u_{h, 0}\right\|^{2}\right] \leq C \quad \text { for } \quad \beta \in[0,2] .
$$

Similarly, one obtains

$$
\mathbb{E}\left[\left\|\Lambda_{h}^{(\beta-1) / 2} S_{h}(t) v_{h, 0}\right\|^{2}\right] \leq C .
$$

To estimate the third term, we use (14), the assumptions on $f$ given in (9), and the equivalence of the norms stated in (15). First, for $\beta \in[0,1]$ we get

$$
\begin{aligned}
& \mathbb{E}\left[\left\|\int_{0}^{t} \Lambda_{h}^{(\beta-1) / 2} S_{h}(t-r) \mathcal{P}_{h} f\left(u_{h, 1}(r)\right) \mathrm{d} r\right\|^{2}\right] \\
& \leq C_{1}+C_{2} \int_{0}^{t} \mathbb{E}\left[\left\|u_{h, 1}(r)\right\|^{2}\right] \mathrm{d} r \\
& \quad \leq C_{3}+C_{4} \int_{0}^{t} \mathbb{E}\left[\left\|u_{h, 1}(r)\right\|_{h, \beta}^{2}\right] \mathrm{d} r
\end{aligned}
$$

Copyright $@$ by SIAM. Unauthorized reproduction of this article is prohibited. 
because $S_{h}(t)$ and $\Lambda_{h}^{-(1-\beta) / 2}$ are bounded. For $\beta \in[1,2]$, we have by (14)

$$
\begin{aligned}
\mathbb{E} & {\left[\left\|\int_{0}^{t} S_{h}(t-r) \Lambda_{h}^{(\beta-1) / 2} \mathcal{P}_{h} \Lambda^{-(\beta-1) / 2} \Lambda^{(\beta-1) / 2} f\left(u_{h, 1}(r)\right) \mathrm{d} r\right\|^{2}\right] } \\
& \leq C \int_{0}^{t} \mathbb{E}\left[1+\left\|\Lambda^{(\beta-1) / 2} u_{h, 1}(r)\right\|^{2}\right] \mathrm{d} r \leq C_{1}+C_{2} \int_{0}^{t} \mathbb{E}\left[\left\|u_{h, 1}(r)\right\|_{h, \beta-1}^{2}\right] \mathrm{d} r \\
& \leq C_{3}+C_{4} \int_{0}^{t} \mathbb{E}\left[\left\|u_{h, 1}(r)\right\|_{h, \beta}^{2}\right] \mathrm{d} r .
\end{aligned}
$$

Finally, Ito's isometry, (15) and (14), and the assumptions (9) on $g$ give us

$$
\begin{array}{r}
\mathbb{E}\left[\left\|\int_{0}^{t} S_{h}(t-r) \mathcal{P}_{h} \Lambda^{-(\beta-1) / 2} \Lambda^{(\beta-1) / 2} g\left(u_{h, 1}(r)\right) \mathrm{d} W(r)\right\|^{2}\right] \\
\leq C_{3}+C_{4} \int_{0}^{t} \mathbb{E}\left[\left\|u_{h, 1}(r)\right\|_{h, \beta}^{2}\right] \mathrm{d} r .
\end{array}
$$

For $\beta \in[0,2]$, we collect all of the above estimates and arrive at

$$
\mathbb{E}\left[\left\|u_{h, 1}(t)\right\|_{h, \beta}^{2}\right] \leq K_{1}+K_{2} \int_{0}^{t} \mathbb{E}\left[\left\|u_{h, 1}(r)\right\|_{h, \beta}^{2}\right] \mathrm{d} r,
$$

and an application of Gronwall's lemma gives the desired bound for $\mathbb{E}\left[\left\|u_{h, 1}(t)\right\|_{h, \beta}^{2}\right]$.

The proof for the other bound is done in the same way except for a slight difference in the initial values and the replacement of $\Lambda_{h}^{(\beta-1) / 2} S_{h}(t-r)$ in the integrals by $\Lambda_{h}^{(\beta-1) / 2} C_{h}(t-r)$.

We now prove a Hölder regularity property of the finite element solution. We write, for $0 \leq s \leq t \leq T$,

$$
\begin{aligned}
u_{h, 1}(t)-u_{h, 1}(s)= & \left(C_{h}(t)-C_{h}(s)\right) u_{h, 0}+\Lambda_{h}^{-1 / 2}\left(S_{h}(t)-S_{h}(s)\right) v_{h, 0} \\
& +\int_{0}^{s} \Lambda_{h}^{-1 / 2}\left(S_{h}(t-r)-S_{h}(s-r)\right) \mathcal{P}_{h} f\left(u_{h, 1}(r)\right) \mathrm{d} r \\
& +\int_{s}^{t} \Lambda_{h}^{-1 / 2} S_{h}(t-r) \mathcal{P}_{h} f\left(u_{h, 1}(r)\right) \mathrm{d} r \\
& +\int_{0}^{s} \Lambda_{h}^{-1 / 2}\left(S_{h}(t-r)-S_{h}(s-r)\right) \mathcal{P}_{h} g\left(u_{h, 1}(r)\right) \mathrm{d} W(r) \\
& +\int_{s}^{t} \Lambda_{h}^{-1 / 2} S_{h}(t-r) \mathcal{P}_{h} g\left(u_{h, 1}(r)\right) \mathrm{d} W(r) .
\end{aligned}
$$

To estimate the first term, we use (12) to get

$$
\begin{aligned}
\mathbb{E}\left[\left\|\left(C_{h}(t)-C_{h}(s)\right) u_{h, 0}\right\|^{2}\right] & =\mathbb{E}\left[\left\|\left(C_{h}(t)-C_{h}(s)\right) \Lambda_{h}^{-\beta / 2} \Lambda_{h}^{\beta / 2} u_{h, 0}\right\|^{2}\right] \\
& \leq C|t-s|^{2 \beta} \mathbb{E}\left[\left\|\Lambda_{h}^{\beta / 2} u_{h, 0}\right\|^{2}\right]
\end{aligned}
$$

for $\beta \in[0,1]$. For $\beta \in(1,2]$ we note that $\Lambda_{h}^{-\beta / 2}=\Lambda_{h}^{-1 / 2} \Lambda_{h}^{-(\beta-1) / 2}$ and that $\Lambda_{h}^{-(\beta-1) / 2}$ is bounded in the operator norm. Using a similar argument for the second term, we get the following estimate for the first two terms:

$$
\begin{aligned}
& \mathbb{E}\left[\left\|\left(C_{h}(t)-C_{h}(s)\right) u_{h, 0}+\Lambda_{h}^{-1 / 2}\left(S_{h}(t)-S_{h}(s)\right) v_{h, 0}\right\|^{2}\right] \\
& \quad \leq C|t-s|^{2 \min (\beta, 1)} \mathbb{E}\left[\left\|u_{h, 0}\right\|_{h, \beta}^{2}+\left\|v_{h, 0}\right\|_{h, \beta-1}^{2}\right]
\end{aligned}
$$

Copyright (c) by SIAM. Unauthorized reproduction of this article is prohibited. 
for $\beta \in[0,2]$. In order to estimate the third term, we use (12), the assumptions on $f$, and the equivalence of the norms given in (15). First, for $\beta \in[0,1]$ we obtain

$$
\begin{aligned}
\mathbb{E}[\| & \left.\int_{0}^{s} \Lambda_{h}^{-1 / 2}\left(S_{h}(t-r)-S_{h}(s-r)\right) \mathcal{P}_{h} f\left(u_{h, 1}(r)\right) \mathrm{d} r \|^{2}\right] \\
& \leq C|t-s|^{2} \sup _{t \in[0, T]} \mathbb{E}\left[1+\left\|u_{h, 1}(t)\right\|^{2}\right] \\
& \leq C|t-s|^{2} \sup _{t \in[0, T]} \mathbb{E}\left[1+\left\|u_{h, 1}(t)\right\|_{h, \beta}^{2}\right] .
\end{aligned}
$$

For $\beta \in[1,2]$ we have, using (12), (14), (15), and the fact that $\Lambda_{h}^{-(\beta-1) / 2}$ is bounded in the operator norm,

$$
\begin{aligned}
\mathbb{E}[\| & \left.\int_{0}^{s} \Lambda_{h}^{-1 / 2}\left(S_{h}(t-r)-S_{h}(s-r)\right) \mathcal{P}_{h} f\left(u_{h, 1}(r)\right) \mathrm{d} r \|^{2}\right] \\
\leq & \int_{0}^{s} \mathbb{E}\left[\|\left(S_{h}(t-r)-S_{h}(s-r)\right) \Lambda_{h}^{-1 / 2} \Lambda_{h}^{-(\beta-1) / 2} \Lambda_{h}^{(\beta-1) / 2} \mathcal{P}_{h} \Lambda^{-(\beta-1) / 2}\right. \\
& \left.\quad \times \Lambda^{(\beta-1) / 2} f\left(u_{h, 1}(r)\right) \|^{2}\right] \mathrm{d} r \\
\leq & C|t-s|^{2} \sup _{t \in[0, T]} \mathbb{E}\left[1+\left\|u_{h, 1}(t)\right\|_{h, \beta-1}^{2}\right] \\
\leq & C|t-s|^{2} \sup _{t \in[0, T]} \mathbb{E}\left[1+\left\|u_{h, 1}(t)\right\|_{h, \beta}^{2}\right] .
\end{aligned}
$$

Similarly, we get for the fourth term

$$
\begin{aligned}
& \mathbb{E}\left[\left\|\int_{s}^{t} \Lambda_{h}^{-1 / 2} S_{h}(t-r) \mathcal{P}_{h} f\left(u_{h, 1}(r)\right) \mathrm{d} r\right\|^{2}\right] \\
& \quad \leq C|t-s|^{2 \min (\beta, 1)} \sup _{t \in[0, T]} \mathbb{E}\left[1+\left\|u_{h, 1}(t)\right\|_{h, \beta}^{2}\right] .
\end{aligned}
$$

To estimate terms five and six, we use Ito's isometry, (12), (14), (15), and the assumptions on $g$ to get, for $\beta \in[0,1]$,

$$
\begin{aligned}
& \mathbb{E}\left[\left\|\int_{0}^{s} \Lambda_{h}^{-1 / 2}\left(S_{h}(t-r)-S_{h}(s-r)\right) \mathcal{P}_{h} g\left(u_{h, 1}(r)\right) \mathrm{d} W(r)\right\|^{2}\right] \\
& \leq \int_{0}^{s} \mathbb{E}\left[\|\left(S_{h}(t-r)-S_{h}(s-r)\right) \Lambda_{h}^{-\beta / 2} \Lambda_{h}^{(\beta-1) / 2} \mathcal{P}_{h}\right. \\
& \left.\quad \times g\left(u_{h, 1}(r)\right) \|_{\mathcal{L}_{2}^{0}}^{2}\right] \mathrm{d} r \\
& \leq C|t-s|^{2 \beta} \sup _{t \in[0, T]} \mathbb{E}\left[\left\|\Lambda_{h}^{(\beta-1) / 2} g\left(u_{h, 1}(t)\right)\right\|_{\mathcal{L}_{2}^{0}}^{2}\right] \\
& \leq C|t-s|^{2 \beta} \sup _{t \in[0, T]} \mathbb{E}\left[1+\left\|u_{h, 1}(t)\right\|_{h, \beta}^{2}\right]
\end{aligned}
$$

Copyright (c) by SIAM. Unauthorized reproduction of this article is prohibited. 
and

$$
\begin{aligned}
\mathbb{E}\left[\left\|\int_{s}^{t} \Lambda_{h}^{-1 / 2} S_{h}(t-r) \mathcal{P}_{h} g\left(u_{h, 1}(r)\right) \mathrm{d} W(r)\right\|^{2}\right] \\
\quad \leq \int_{s}^{t} \mathbb{E}\left[\left\|S_{h}(t-r) \Lambda_{h}^{-\beta / 2} \Lambda_{h}^{(\beta-1) / 2} \mathcal{P}_{h} g\left(u_{h, 1}(r)\right)\right\|_{\mathcal{L}_{2}^{0}}^{2}\right] \mathrm{d} r \\
\quad \leq C|t-s|^{2 \beta} \sup _{t \in[0, T]} \mathbb{E}\left[\left\|\Lambda_{h}^{(\beta-1) / 2} g\left(u_{h, 1}(t)\right)\right\|_{\mathcal{L}_{2}^{0}}^{2}\right] \\
\leq C|t-s|^{2 \beta} \sup _{t \in[0, T]} \mathbb{E}\left[1+\left\|u_{h, 1}(t)\right\|_{h, \beta}^{2}\right] .
\end{aligned}
$$

For $\beta \in[1,2]$ we again use that $\Lambda_{h}^{-(\beta-1) / 2}$ is bounded in the operator norm.

Collecting the above estimates gives us the statement about the regularity of the finite element solution.

3. Mean-square convergence analysis. Recall that the exact solutions to (2) and (4) solve the equations

$$
\begin{aligned}
X(t) & =E(t) X_{0}+\int_{0}^{t} E(t-s) F(X(s)) \mathrm{d} s+\int_{0}^{t} E(t-s) G(X(s)) \mathrm{d} W(s), \\
X_{h}(t) & =E_{h}(t) X_{h, 0}+\int_{0}^{t} E_{h}(t-s) \mathcal{P}_{h} F\left(X_{h}(s)\right) \mathrm{d} s+\int_{0}^{t} E_{h}(t-s) \mathcal{P}_{h} G\left(X_{h}(s)\right) \mathrm{d} W(s),
\end{aligned}
$$

where

$$
X_{0}=\left[\begin{array}{l}
u_{0} \\
v_{0}
\end{array}\right], \quad X_{h, 0}=\left[\begin{array}{l}
\mathcal{R}_{h} u_{0} \\
\mathcal{P}_{h} v_{0}
\end{array}\right]
$$

and

$$
E(t)=\left[\begin{array}{cc}
C(t) & \Lambda^{-1 / 2} S(t) \\
-\Lambda^{1 / 2} S(t) & C(t)
\end{array}\right], \quad E_{h}(t)=\left[\begin{array}{cc}
C_{h}(t) & \Lambda_{h}^{-1 / 2} S_{h}(t) \\
-\Lambda_{h}^{1 / 2} S_{h}(t) & C_{h}(t)
\end{array}\right]
$$

with $C(t)=\cos \left(t \Lambda^{1 / 2}\right), S(t)=\sin \left(t \Lambda^{1 / 2}\right), C_{h}(t)=\cos \left(t \Lambda_{h}^{1 / 2}\right)$, and $S_{h}(t)=\sin \left(t \Lambda_{h}^{1 / 2}\right)$.

The explicit time discretization of the finite element solution (4) of the stochastic wave equation using a stochastic trigonometric method with step size $k$ reads

$$
U^{n+1}=E_{h}(k) U^{n}+E_{h}(k) \mathcal{P}_{h} F\left(U^{n}\right) k+E_{h}(k) \mathcal{P}_{h} G\left(U^{n}\right) \Delta W^{n}
$$

that is,

$$
\begin{aligned}
{\left[\begin{array}{c}
U_{1}^{n+1} \\
U_{2}^{n+1}
\end{array}\right]=} & {\left[\begin{array}{cc}
C_{h}(k) & \Lambda_{h}^{-1 / 2} S_{h}(k) \\
-\Lambda_{h}^{1 / 2} S_{h}(k) & C_{h}(k)
\end{array}\right]\left[\begin{array}{c}
U_{1}^{n} \\
U_{2}^{n}
\end{array}\right]+\left[\begin{array}{c}
\Lambda_{h}^{-1 / 2} S_{h}(k) \\
C_{h}(k)
\end{array}\right] \mathcal{P}_{h} f\left(U_{1}^{n}\right) k } \\
& +\left[\begin{array}{c}
\Lambda_{h}^{-1 / 2} S_{h}(k) \\
C_{h}(k)
\end{array}\right] \mathcal{P}_{h} g\left(U_{1}^{n}\right) \Delta W^{n}
\end{aligned}
$$

where $\Delta W^{n}=W\left(t_{n+1}\right)-W\left(t_{n}\right)$ denotes the Wiener increments. Here we thus get an approximation $U_{j}^{n} \approx u_{h, j}\left(t_{n}\right)$ of the exact solution of our finite element problem at the discrete times $t_{n}=n k$. Further, a recursion gives

$$
U^{n}=E_{h}\left(t_{n}\right) U^{0}+\sum_{j=0}^{n-1} E_{h}\left(t_{n}-t_{j}\right) \mathcal{P}_{h} F\left(U^{j}\right) k+\sum_{j=0}^{n-1} E_{h}\left(t_{n}-t_{j}\right) \mathcal{P}_{h} G\left(U^{j}\right) \Delta W^{j}
$$

Copyright (C) by SIAM. Unauthorized reproduction of this article is prohibited. 
We now look at the error between the numerical and the exact solutions $U^{n}-X\left(t_{n}\right)$. We follow the same approach as in [23] for parabolic problems (see also [17]) and obtain

$$
\mathbb{E}\left[\left\|U^{n}-X\left(t_{n}\right)\right\|^{2}\right] \leq 3\left(\mathbb{E}\left[\left\|\operatorname{Err}_{0}\right\|^{2}\right]+\mathbb{E}\left[\left\|\operatorname{Err}_{\mathrm{d}}\right\|^{2}\right]+\mathbb{E}\left[\left\|\operatorname{Err}_{\mathrm{s}}\right\|^{2}\right]\right),
$$

where we define

$$
\begin{gathered}
\operatorname{Err}_{0}:=\left(E_{h}\left(t_{n}\right) \mathcal{P}_{h}-E\left(t_{n}\right)\right) X_{0} \\
\operatorname{Err}_{\mathrm{d}}:=\sum_{j=0}^{n-1} \int_{t_{j}}^{t_{j+1}}\left(E_{h}\left(t_{n}-t_{j}\right) \mathcal{P}_{h} F\left(U^{j}\right)-E\left(t_{n}-s\right) F(X(s))\right) \mathrm{d} s
\end{gathered}
$$

and

$$
\operatorname{Err}_{\mathrm{s}}:=\sum_{j=0}^{n-1} \int_{t_{j}}^{t_{j+1}}\left(E_{h}\left(t_{n}-t_{j}\right) \mathcal{P}_{h} G\left(U^{j}\right)-E\left(t_{n}-s\right) G(X(s))\right) \mathrm{d} W(s) .
$$

We next estimate the above three terms.

Estimate for the initial error $\mathbf{E r r}_{\mathbf{0}}$. By (10), the first component reads

$$
\begin{aligned}
& \mathbb{E}\left[\left\|\left(C_{h}\left(t_{n}\right) \mathcal{R}_{h}-C\left(t_{n}\right)\right) u_{0}+\left(\Lambda_{h}^{-1 / 2} S_{h}\left(t_{n}\right) \mathcal{P}_{h}-\Lambda^{-1 / 2} S\left(t_{n}\right)\right) v_{0}\right\|^{2}\right] \\
& \quad \leq C\left(1+t_{n}\right)^{2} h^{2(\gamma-1)}\left(\mathbb{E}\left[\left\|u_{0}\right\|_{\gamma}+\left\|v_{0}\right\|_{\gamma-1}\right]\right)^{2}
\end{aligned}
$$

for $\gamma \in[1,3]$. Similarly, for the second component

$$
\begin{aligned}
& \mathbb{E}\left[\left\|-\left(\Lambda_{h}^{1 / 2} S_{h}\left(t_{n}\right) \mathcal{R}_{h}-\Lambda^{1 / 2} S\left(t_{n}\right)\right) u_{0}+\left(C_{h}\left(t_{n}\right) \mathcal{P}_{h}-C\left(t_{n}\right)\right) v_{0}\right\|^{2}\right] \\
& \quad \leq C\left(1+t_{n}\right)^{2} h^{\frac{4}{3}(\gamma-1)}\left(\mathbb{E}\left[\left\|u_{0}\right\|_{\gamma}+\left\|v_{0}\right\|_{\gamma-1}\right]\right)^{2}
\end{aligned}
$$

for $\gamma \in[1,4]$.

Estimate for the deterministic part $\mathbf{E r r}_{\mathbf{d}}$. We write the deterministic error as

$$
\begin{aligned}
\operatorname{Err}_{\mathrm{d}}= & \sum_{j=0}^{n-1} \int_{t_{j}}^{t_{j+1}}\left(E_{h}\left(t_{n}-t_{j}\right) \mathcal{P}_{h} F\left(U^{j}\right)-E\left(t_{n}-s\right) F(X(s))\right) \mathrm{d} s \\
= & \sum_{j=0}^{n-1} \int_{t_{j}}^{t_{j+1}} E_{h}\left(t_{n}-t_{j}\right) \mathcal{P}_{h}\left(F\left(U^{j}\right)-F\left(X\left(t_{j}\right)\right)\right) \mathrm{d} s \\
& +\sum_{j=0}^{n-1} \int_{t_{j}}^{t_{j+1}} E_{h}\left(t_{n}-t_{j}\right) \mathcal{P}_{h}\left(F\left(X\left(t_{j}\right)\right)-F(X(s))\right) \mathrm{d} s \\
& +\sum_{j=0}^{n-1} \int_{t_{j}}^{t_{j+1}}\left(E_{h}\left(t_{n}-t_{j}\right) \mathcal{P}_{h}-E\left(t_{n}-t_{j}\right)\right) F(X(s)) \mathrm{d} s \\
& +\sum_{j=0}^{n-1} \int_{t_{j}}^{t_{j+1}}\left(E\left(t_{n}-t_{j}\right)-E\left(t_{n}-s\right)\right) F(X(s)) \mathrm{d} s \\
= & I_{1}+I_{2}+I_{3}+I_{4}
\end{aligned}
$$

Copyright $@$ by SIAM. Unauthorized reproduction of this article is prohibited. 
and estimate the second moment of each term in the above equation. For the first component of the first term, we get the following estimate by using (13) and (9):

$$
\begin{aligned}
\left(\mathbb{E}\left[\left\|I_{[1,1]}\right\|^{2}\right]\right)^{1 / 2} & \leq \sum_{j=0}^{n-1} \int_{t_{j}}^{t_{j+1}}\left(\mathbb{E}\left[\left\|\Lambda_{h}^{-1 / 2} S_{h}\left(t_{n}-t_{j}\right) \mathcal{P}_{h}\left(f\left(U_{1}^{j}\right)-f\left(u_{1}\left(t_{j}\right)\right)\right)\right\|^{2}\right]\right)^{1 / 2} \mathrm{~d} s \\
& \leq C \sum_{j=0}^{n-1} k\left(\mathbb{E}\left[\left\|U_{1}^{j}-u\left(t_{j}\right)\right\|^{2}\right]\right)^{1 / 2},
\end{aligned}
$$

so that

$$
\mathbb{E}\left[\left\|I_{[1,1]}\right\|^{2}\right] \leq\left(C k \sum_{j=0}^{n-1}\left(\mathbb{E}\left[\left\|U_{1}^{j}-u\left(t_{j}\right)\right\|^{2}\right]\right)^{1 / 2}\right)^{2} \leq C k \sum_{j=0}^{n-1} \mathbb{E}\left[\left\|U_{1}^{j}-u\left(t_{j}\right)\right\|^{2}\right]
$$

The second component is estimated in the same way:

$$
\begin{aligned}
\left(\mathbb{E}\left[\left\|I_{[1,2]}\right\|^{2}\right]\right)^{1 / 2} & \leq \sum_{j=0}^{n-1} \int_{t_{j}}^{t_{j+1}}\left(\mathbb{E}\left[\left\|C_{h}\left(t_{n}-t_{j}\right) \mathcal{P}_{h}\left(f\left(U_{1}^{j}\right)-f\left(u_{1}\left(t_{j}\right)\right)\right)\right\|^{2}\right]\right)^{1 / 2} \mathrm{~d} s \\
& \leq C \sum_{j=0}^{n-1} k\left(\mathbb{E}\left[\left\|U_{1}^{j}-u\left(t_{j}\right)\right\|^{2}\right]\right)^{1 / 2} .
\end{aligned}
$$

For the second term, using Proposition 2, we get

$$
\begin{aligned}
& \left(\mathbb{E}\left[\left\|I_{[2,1]}\right\|^{2}\right]\right)^{1 / 2} \\
& \leq \sum_{j=0}^{n-1} \int_{t_{j}}^{t_{j+1}}\left(\mathbb{E}\left[\left\|\Lambda_{h}^{-1 / 2} S_{h}\left(t_{n}-t_{j}\right) \mathcal{P}_{h}\left(f\left(u_{1}\left(t_{j}\right)\right)-f\left(u_{1}(s)\right)\right)\right\|^{2}\right]\right)^{1 / 2} \mathrm{~d} s \\
& \leq C \sum_{j=0}^{n-1} \int_{t_{j}}^{t_{j+1}}\left(\mathbb{E}\left[\left\|u_{1}\left(t_{j}\right)-u_{1}(s)\right\|^{2}\right]\right)^{1 / 2} \mathrm{~d} s \\
& \leq C \sum_{j=0}^{n-1} \int_{t_{j}}^{t_{j+1}}\left|t_{j}-s\right|^{\min (\beta, 1)} \mathrm{d} s\left(\mathbb{E}\left[\left\|u_{0}\right\|_{\beta}^{2}+\left\|v_{0}\right\|_{\beta-1}^{2}\right]+\sup _{t \in[0, T]} \mathbb{E}\left[1+\left\|u_{1}(t)\right\|_{\beta}^{2}\right]\right)^{1 / 2} \\
& \leq C k^{\min (\beta, 1)}
\end{aligned}
$$

for $\beta \in[0,3]$. Thus,

$$
\mathbb{E}\left[\left\|I_{[2,1]}\right\|^{2}\right] \leq C k^{2 \min (\beta, 1)} .
$$

The second component $I_{[2,2]}$ has the same expression as $I_{[1,2]}$ except that $\Lambda_{h}^{-1 / 2} S_{h}\left(t_{n}-\right.$ $\left.t_{j}\right)$ is replaced by $C_{h}\left(t_{n}-t_{j}\right)$. The same estimate holds since the cosine operator is bounded.

Copyright $@$ ㅇ by SIAM. Unauthorized reproduction of this article is prohibited. 
The third term reads as follows, using $\mathcal{K}_{h}(t)$ in (11) and $\beta \in[1,3]$ :

$$
\begin{aligned}
\left(\mathbb{E}\left[\left\|I_{[3,1]}\right\|^{2}\right]\right)^{1 / 2} & \leq \sum_{j=0}^{n-1} \int_{t_{j}}^{t_{j+1}}\left(\mathbb{E}\left[\left\|\left(\Lambda_{h}^{-1 / 2} S_{h}\left(t_{n}-t_{j}\right) \mathcal{P}_{h}-\Lambda^{-1 / 2} S\left(t_{n}-t_{j}\right)\right) f\left(u_{1}(s)\right)\right\|^{2}\right]\right)^{1 / 2} \mathrm{~d} s \\
& =\sum_{j=0}^{n-1} \int_{t_{j}}^{t_{j+1}}\left(\mathbb{E}\left[\left\|\mathcal{K}_{h}\left(t_{n}-t_{j}\right) f\left(u_{1}(s)\right)\right\|^{2}\right]\right)^{1 / 2} \mathrm{~d} s \\
& \leq C h^{\frac{2}{3} \beta} \sum_{j=0}^{n-1} \int_{t_{j}}^{t_{j+1}}\left(\mathbb{E}\left[\left\|\Lambda^{(\beta-1) / 2} f\left(u_{1}(s)\right)\right\|^{2}\right]\right)^{1 / 2} \mathrm{~d} s \\
& \leq C h^{\frac{2}{3} \beta}\left(\sup _{t \in[0, T]} \mathbb{E}\left[1+\left\|u_{1}(t)\right\|_{\beta-1}^{2}\right]\right)^{1 / 2} \\
& \leq C h^{\frac{2}{3} \beta} .
\end{aligned}
$$

For $\beta \in[0,1]$ we simply note that

$$
\mathbb{E}\left[\left\|\Lambda^{(\beta-1) / 2} f\left(u_{1}(s)\right)\right\|^{2}\right] \leq C \mathbb{E}\left[\left\|f\left(u_{1}(s)\right)\right\|^{2}\right] \leq C .
$$

The estimate for the second component is done in a similar way, now using $\dot{\mathcal{K}}_{h}(t)$ in (11) with $\beta \in[1,4]$ :

$$
\mathbb{E}\left[\left\|I_{[3,2]}\right\|^{2}\right] \leq C h^{\frac{4}{3}(\beta-1)} .
$$

For the fourth term with $\beta \in[0,3]$, using (13) and the assumption on the function $f$ in (9), we get

$$
\begin{aligned}
\left(\mathbb{E}\left[\left\|I_{[4,1]}\right\|^{2}\right]\right)^{1 / 2} & \leq \sum_{j=0}^{n-1} \int_{t_{j}}^{t_{j+1}}\left(\mathbb{E}\left[\left\|\left(S\left(t_{n}-t_{j}\right)-S\left(t_{n}-s\right)\right) \Lambda^{-1 / 2} f\left(u_{1}(s)\right)\right\|^{2}\right]\right)^{1 / 2} \mathrm{~d} s \\
& \leq C \sum_{j=0}^{n-1} \int_{t_{j}}^{t_{j+1}}\left(\left\|\left(S\left(t_{n}-t_{j}\right)-S\left(t_{n}-s\right)\right) \Lambda^{-1 / 2}\right\|_{\mathcal{L}(U)}^{2} \mathbb{E}\left[\left\|f\left(u_{1}(s)\right)\right\|^{2}\right]\right)^{1 / 2} \mathrm{~d} s \\
& \leq C \sum_{j=0}^{n-1} \int_{t_{j}}^{t_{j+1}}\left(\left|s-t_{j}\right|^{2} \mathbb{E}\left[1+\left\|u_{1}(s)\right\|^{2}\right]\right)^{1 / 2} \mathrm{~d} s \\
& \leq C k .
\end{aligned}
$$

Thus, we obtain

$$
\mathbb{E}\left[\left\|I_{[4,1]}\right\|^{2}\right] \leq C k^{2}
$$

Copyright $@$ by SIAM. Unauthorized reproduction of this article is prohibited. 
For the second component we get

$$
\begin{aligned}
&\left(\mathbb{E}\left[\left\|I_{[4,2]}\right\|^{2}\right]\right)^{1 / 2} \leq \sum_{j=0}^{n-1} \int_{t_{j}}^{t_{j+1}}\left(\mathbb{E}\left[\left\|\left(C\left(t_{n}-t_{j}\right)-C\left(t_{n}-s\right)\right) f\left(u_{1}(s)\right)\right\|^{2}\right]\right)^{1 / 2} \mathrm{~d} s \\
& \leq C \sum_{j=0}^{n-1} \int_{t_{j}}^{t_{j+1}}\left(\left\|\left(C\left(t_{n}-t_{j}\right)-C\left(t_{n}-s\right)\right) \Lambda^{-(\beta-1) / 2}\right\|_{\mathcal{L}(U)}^{2}\right. \\
&\left.\times \mathbb{E}\left[\left\|\Lambda^{(\beta-1) / 2} f\left(u_{1}(s)\right)\right\|^{2}\right]\right)^{1 / 2} \mathrm{~d} s \\
& \leq C \sum_{j=0}^{n-1} \int_{t_{j}}^{t_{j+1}}\left(\left|s-t_{j}\right|^{2(\beta-1)} \mathbb{E}\left[1+\left\|u_{1}(s)\right\|_{\beta-1}^{2}\right]\right)^{1 / 2} \mathrm{~d} s \\
& \leq C k^{\min (\beta-1,1)}
\end{aligned}
$$

for $\beta \geq 1$.

All together, we thus obtain

$\mathbb{E}\left[\left\|\operatorname{Err}_{\mathrm{d}, 1}\right\|^{2}\right] \leq C \cdot\left(h^{\frac{4 \beta}{3}}+k^{2 \min (\beta, 1)}+k \sum_{j=0}^{n-1} \mathbb{E}\left[\left\|U_{1}^{j}-u_{1}\left(t_{j}\right)\right\|^{2}\right]\right) \quad$ for $\beta \in[0,3]$,

$\mathbb{E}\left[\left\|\operatorname{Err}_{\mathrm{d}, 2}\right\|^{2}\right] \leq C \cdot\left(h^{\frac{4(\beta-1)}{3}}+k^{2 \min (\beta-1,1)}+k \sum_{j=0}^{n-1} \mathbb{E}\left[\left\|U_{1}^{j}-u_{1}\left(t_{j}\right)\right\|^{2}\right]\right) \quad$ for $\beta \in[1,4]$.

Estimate for the stochastic part $\mathbf{E r r}_{\mathbf{s}}$. We rewrite the stochastic part as we did for the deterministic part of the error:

$$
\begin{aligned}
\operatorname{Err}_{\mathrm{s}}= & \sum_{j=0}^{n-1} \int_{t_{j}}^{t_{j+1}}\left(E_{h}\left(t_{n}-t_{j}\right) \mathcal{P}_{h} G\left(U^{j}\right)-E\left(t_{n}-s\right) G(X(s))\right) \mathrm{d} W(s) \\
= & \sum_{j=0}^{n-1} \int_{t_{j}}^{t_{j+1}} E_{h}\left(t_{n}-t_{j}\right) \mathcal{P}_{h}\left(G\left(U^{j}\right)-G\left(X\left(t_{j}\right)\right)\right) \mathrm{d} W(s) \\
& +\sum_{j=0}^{n-1} \int_{t_{j}}^{t_{j+1}} E_{h}\left(t_{n}-t_{j}\right) \mathcal{P}_{h}\left(G\left(X\left(t_{j}\right)\right)-G(X(s))\right) \mathrm{d} W(s) \\
& +\sum_{j=0}^{n-1} \int_{t_{j}}^{t_{j+1}}\left(E_{h}\left(t_{n}-t_{j}\right) \mathcal{P}_{h}-E\left(t_{n}-t_{j}\right)\right) G(X(s)) \mathrm{d} W(s) \\
& +\sum_{j=0}^{n-1} \int_{t_{j}}^{t_{j+1}}\left(E\left(t_{n}-t_{j}\right)-E\left(t_{n}-s\right)\right) G(X(s)) \mathrm{d} W(s) \\
= & J_{1}+J_{2}+J_{3}+J_{4} .
\end{aligned}
$$

The estimate for the first term follows by using the Ito isometry, the boundedness of $\mathcal{P}_{h}, S_{h}$, and $\Lambda_{h}^{-1 / 2}$, and the Lipschitz condition on the function $g$ in (9),

$$
\begin{aligned}
\mathbb{E}\left[\left\|J_{[1,1]}\right\|^{2}\right] & =\sum_{j=0}^{n-1} \int_{t_{j}}^{t_{j+1}} \mathbb{E}\left[\left\|\Lambda_{h}^{-1 / 2} S_{h}\left(t_{n}-t_{j}\right) \mathcal{P}_{h}\left(g\left(U_{1}^{j}\right)-g\left(u_{1}\left(t_{j}\right)\right)\right)\right\|_{\mathcal{L}_{2}^{0}}^{2}\right] \mathrm{d} s \\
& \leq C k \sum_{j=0}^{n-1} \mathbb{E}\left[\left\|U_{1}^{j}-u_{1}\left(t_{j}\right)\right\|^{2}\right]
\end{aligned}
$$

Copyright $@$ by SIAM. Unauthorized reproduction of this article is prohibited. 
for $\beta \in[0,3]$. The same estimate holds for the second component $J_{[1,2]}$ with $\beta \in[1,4]$. For the first component of the second term, using Proposition 2, we obtain

$$
\begin{aligned}
\mathbb{E}\left[\left\|J_{[2,1]}\right\|^{2}\right] & =\sum_{j=0}^{n-1} \int_{t_{j}}^{t_{j+1}} \mathbb{E}\left[\left\|\Lambda_{h}^{-1 / 2} S_{h}\left(t_{n}-t_{j}\right) \mathcal{P}_{h}\left(g\left(u_{1}\left(t_{j}\right)\right)-g\left(u_{1}(s)\right)\right)\right\|_{\mathcal{L}_{2}^{0}}^{2}\right] \mathrm{d} s \\
\leq & C \sum_{j=0}^{n-1} \int_{t_{j}}^{t_{j+1}} \mathbb{E}\left[\left\|u_{1}\left(t_{j}\right)-u_{1}(s)\right\|^{2}\right] \mathrm{d} s \\
\leq C \sum_{j=0}^{n-1} \int_{t_{j}}^{t_{j+1}}\left|t_{j}-s\right|^{2 \min (\beta, 1)} \mathrm{d} s\left(\mathbb{E}\left[\left\|u_{0}\right\|_{\beta}^{2}+\left\|v_{0}\right\|_{\beta-1}^{2}\right]\right. & \left.+\sup _{t \in[0, T]} \mathbb{E}\left[1+\left\|u_{1}(t)\right\|_{\beta}^{2}\right]\right) \\
\leq C k^{2 \min (\beta, 1)} &
\end{aligned}
$$

for $\beta \in[0,3]$. Similarly, the estimate for the second component of $J_{2}$ reads as

$$
\begin{aligned}
\mathbb{E}\left[\left\|J_{[2,2]}\right\|^{2}\right] & =\sum_{j=0}^{n-1} \int_{t_{j}}^{t_{j+1}} \mathbb{E}\left[\left\|C_{h}\left(t_{n}-t_{j}\right) \mathcal{P}_{h}\left(g\left(u_{1}\left(t_{j}\right)\right)-g\left(u_{1}(s)\right)\right)\right\|_{\mathcal{L}_{2}^{0}}^{2}\right] \mathrm{d} s \\
& \leq C k^{2}\left(\mathbb{E}\left[\left\|u_{0}\right\|_{\beta}^{2}+\left\|v_{0}\right\|_{\beta-1}^{2}\right]+\sup _{t \in[0, T]} \mathbb{E}\left[1+\left\|u_{1}(t)\right\|_{\beta}^{2}\right]\right) .
\end{aligned}
$$

For the second component we have $\beta \in[1,4]$, so that $\min (\beta, 1)=1$. For the first component of the third term we use (11) with $\beta \in(1,3]$ to get

$$
\begin{aligned}
\mathbb{E}\left[\left\|J_{[3,1]}\right\|^{2}\right] & =\sum_{j=0}^{n-1} \int_{t_{j}}^{t_{j+1}} \mathbb{E}\left[\left\|\left(\Lambda_{h}^{-1 / 2} S_{h}\left(t_{n}-t_{j}\right) \mathcal{P}_{h}-\Lambda^{-1 / 2} S\left(t_{n}-t_{j}\right)\right) g\left(u_{1}(s)\right)\right\|_{\mathcal{L}_{2}^{0}}^{2}\right] \mathrm{d} s \\
& \leq C h^{\frac{4}{3} \beta} \sum_{j=0}^{n-1} \int_{t_{j}}^{t_{j+1}} \mathbb{E}\left[\left\|\Lambda^{(\beta-1) / 2} g\left(u_{1}(s)\right)\right\|^{2}\right] \mathrm{d} s \\
& \leq C h^{\frac{4 \beta}{3}} \sup _{t \in[0, T]} \mathbb{E}\left[1+\left\|u_{1}(t)\right\|_{\beta}^{2}\right] \leq C h^{\frac{4 \beta}{3}}
\end{aligned}
$$

by Proposition 2. The estimate for $\beta \in[0,1]$ is obtained in the same way. For the second component, we also obtain

$$
\mathbb{E}\left[\left\|J_{[3,2]}\right\|^{2}\right] \leq C h^{\frac{4(\beta-1)}{3}} \sup _{t \in[0, T]} \mathbb{E}\left[1+\left\|u_{1}(t)\right\|_{\beta}^{2}\right] \leq C h^{\frac{4(\beta-1)}{3}}
$$

for $\beta \in[1,4]$. Finally, for the first component of the fourth term, we get

$$
\begin{aligned}
\mathbb{E}\left[\left\|J_{[4,1]}\right\|^{2}\right] & =\sum_{j=0}^{n-1} \int_{t_{j}}^{t_{j+1}} \mathbb{E}\left[\left\|\left(S\left(t_{n}-t_{j}\right)-S\left(t_{n}-s\right)\right) \Lambda^{-\beta / 2} \Lambda^{(\beta-1) / 2} g\left(u_{1}(s)\right)\right\|_{\mathcal{L}_{2}^{0}}^{2}\right] \mathrm{d} s \\
& \leq C \sum_{j=0}^{n-1} \int_{t_{j}}^{t_{j+1}}\left|s-t_{j}\right|^{2 \beta} \mathrm{d} s \sup _{t \in[0, T]} \mathbb{E}\left[1+\left\|u_{1}(t)\right\|^{2}\right] \\
& \leq C k^{2 \beta} \sup _{t \in[0, T]} \mathbb{E}\left[1+\left\|u_{1}(t)\right\|^{2}\right]
\end{aligned}
$$

Copyright $@$ by SIAM. Unauthorized reproduction of this article is prohibited. 
for $\beta \in[0,1]$. For $\beta>1$, we note that $\Lambda^{-\beta / 2}=\Lambda^{-1 / 2} \Lambda^{-(\beta-1) / 2}$ and that $\Lambda^{-(\beta-1) / 2}$ is bounded so that we get

$$
\mathbb{E}\left[\left\|J_{[4,1]}\right\|^{2}\right] \leq C k^{2 \min (\beta, 1)} .
$$

Similarly, for the second component, using the regularity of the cosine operator, we obtain

$$
\mathbb{E}\left[\left\|J_{[4,2]}\right\|^{2}\right] \leq C k^{2 \min (\beta-1,1)}
$$

for $\beta \geq 1$. The estimate for the stochastic error thus reads

$$
\begin{aligned}
& \mathbb{E}\left[\left\|\operatorname{Err}_{\mathrm{s}, 1}\right\|^{2}\right] \leq C \cdot\left(h^{\frac{4 \beta}{3}}+k^{2 \min (\beta, 1)}+k \sum_{j=0}^{n-1} \mathbb{E}\left[\left\|U_{1}^{j}-u_{1}\left(t_{j}\right)\right\|^{2}\right]\right) \quad \text { for } \beta \in[0,3], \\
& \mathbb{E}\left[\left\|\operatorname{Err}_{\mathrm{s}, 2}\right\|^{2}\right] \leq C \cdot\left(h^{\frac{4(\beta-1)}{3}}+k^{2 \min (\beta-1,1)}+k \sum_{j=0}^{n-1} \mathbb{E}\left[\left\|U_{1}^{j}-u_{1}\left(t_{j}\right)\right\|^{2}\right]\right) \quad \text { for } \beta \in[1,4] .
\end{aligned}
$$

Collecting the estimates of the three parts of the error, we thus obtain the following estimate for the error in the position and velocity of the stochastic wave equation:

$$
\begin{aligned}
& \mathbb{E}\left[\left\|U_{1}^{n}-u_{1}\left(t_{n}\right)\right\|^{2}\right] \leq C \cdot\left(h^{\frac{4 \beta}{3}}+k^{2 \min (\beta, 1)}+k \sum_{j=0}^{n-1} \mathbb{E}\left[\left\|U_{1}^{j}-u_{1}\left(t_{j}\right)\right\|^{2}\right]\right), \quad \beta \in[0,3], \\
& \mathbb{E}\left[\left\|U_{2}^{n}-u_{2}\left(t_{n}\right)\right\|^{2}\right] \leq C \cdot\left(h^{\frac{4}{3}(\beta-1)}+k^{2 \min (\beta-1,1)}+k \sum_{j=0}^{n-1} \mathbb{E}\left[\left\|U_{1}^{j}-u_{1}\left(t_{j}\right)\right\|^{2}\right]\right), \beta \in[1,4] .
\end{aligned}
$$

Using the above error bounds and an application of the discrete Gronwall lemma proves the following result for the mean-square errors of the full discretization of the semilinear stochastic wave equation with a multiplicative noise. We assume that $\gamma$ is large enough so that the stochastic error dominates over the initial error.

THEOREM 4. Consider the numerical discretization of the semilinear stochastic wave equation with a multiplicative noise (1) on a compact time interval $[0, T], T>0$, by a linear finite element method in space and the stochastic trigonometric method (16) in time. Assume that $u_{0} \in L_{2}\left(\Omega, \dot{H}^{\gamma}\right), v_{0} \in L_{2}\left(\Omega, \dot{H}^{\gamma-1}\right)$ with $\gamma \geq 1+2 \beta / 3$, and the functions $f$ and $g$ satisfy (9) for some $\beta \geq 0$ for the error in the position (and for some $\beta \geq 1$ for the error in the velocity). Then, for $t_{n} \in[0, T]$, the mean-square errors read as

$$
\begin{aligned}
\left\|U_{1}^{n}-u_{h, 1}\left(t_{n}\right)\right\|_{L_{2}\left(\Omega, \dot{H}^{0}\right)} & \leq C \cdot k^{\min (\beta, 1)} \quad \text { for } \beta \in[0,2], \\
\left\|U_{2}^{n}-u_{h, 2}\left(t_{n}\right)\right\|_{L_{2}\left(\Omega, \dot{H}^{0}\right)} & \leq C \cdot k^{\min (\beta-1,1)} \quad \text { for } \beta \in[1,2], \\
\left\|U_{1}^{n}-u_{1}\left(t_{n}\right)\right\|_{L_{2}\left(\Omega, \dot{H}^{0}\right)} & \leq C \cdot\left(h^{\frac{2 \beta}{3}}+k^{\min (\beta, 1)}\right) \quad \text { for } \beta \in[0,3], \\
\left\|U_{2}^{n}-u_{2}\left(t_{n}\right)\right\|_{L_{2}\left(\Omega, \dot{H}^{0}\right)} & \leq C \cdot\left(h^{\frac{2(\beta-1)}{3}}+k^{\min (\beta-1,1)}\right) \quad \text { for } \beta \in[1,4] .
\end{aligned}
$$

Observe that the error estimates between the finite element solutions and the solutions given by the stochastic trigonometric method are proven in a similar way as the estimates above, using in addition Proposition 3. 
4. A trace formula. In this section, we will only consider the problem (1) with additive noise $(g \equiv 1$ in $(1))$ and the nonlinearity $f(u)=-V^{\prime}(u)$ for a smooth potential $V$. We will further consider a trace-class $Q$-Wiener process $W$, i.e., $\operatorname{Tr}(Q)=$ $\left\|Q^{1 / 2}\right\|_{\mathrm{HS}}^{2}<\infty$. In this situation, the exact solution of our nonlinear stochastic wave equation satisfies a trace formula (see, for example, $[2,5]$ for linear stochastic wave equations) where, in analogy to deterministic problems, the "Hamiltonian" function is defined on $H^{1}=\dot{H}^{1} \times \dot{H}^{0}$ as

$$
\begin{aligned}
H(X) & =\frac{1}{2} \int_{\mathcal{D}}\left(\left|u_{2}\right|^{2}+\left|\nabla u_{1}\right|^{2}\right) \mathrm{d} x+\int_{\mathcal{D}} V\left(u_{1}\right) \mathrm{d} x \\
& =\frac{1}{2}\left\|u_{2}\right\|^{2}+\frac{1}{2}\left\|\Lambda^{1 / 2} u_{1}\right\|^{2}+\int_{\mathcal{D}} V\left(u_{1}\right) \mathrm{d} x .
\end{aligned}
$$

In this section, we restrict our attention to additive noises, since, in this case, we obtain an elegant and tractable expression for the drift term in the trace formulas (see below). This is not the case for multiplicative noise as explained in the remark following the proof of Proposition 5.

The trace formula for the exact solution to our stochastic wave equation is given in the following proposition.

Proposition 5. Consider the nonlinear stochastic wave equation (1) with additive noise, that is, with $g \equiv 1$. Further, let $f(u)=-V^{\prime}(u)$ for a smooth potential $V$, let $\{W(t)\}_{t \geq 0}$ be a trace-class $Q$-Wiener process, and let the Hamiltonian $H$ be defined as above. Then the exact solution, $X(t)$ in (5), of the nonlinear stochastic wave equation (1), satisfies the trace formula

$$
\mathbb{E}[H(X(t))]=\mathbb{E}[H(X(0))]+t \frac{1}{2} \operatorname{Tr}(Q), \quad t \geq 0 .
$$

Proof. Indeed, using Ito's formula (one can apply Theorem 4.17 in [8] since $X(t)$ is an Ito process and the potential $V$ is smooth enough) for the above Hamiltonian, we obtain

$$
\begin{aligned}
H(X(t))= & H(X(0))+\int_{0}^{t}\left(H^{\prime}(X(s)), G \mathrm{~d} W(s)\right)+\int_{0}^{t}\left(H^{\prime}(X(s)), A X+F(X)\right) \mathrm{d} s \\
& +\frac{1}{2} \int_{0}^{t} \operatorname{Tr}\left[H^{\prime \prime}(X(s))\left(G Q^{1 / 2}\right)\left(G Q^{1 / 2}\right)^{*}\right] \mathrm{d} s
\end{aligned}
$$

for all time $t$. Here we have $G=\left[\begin{array}{l}0 \\ I\end{array}\right]$, since we are concerned with additive noise. The expected value of the second term in the above formula is seen to be zero. Using the definitions of $A$ and the nonlinearity $F$, the integrand present in the third term reads as

$$
\left(\Lambda u_{1}, u_{2}\right)+\left(V^{\prime}\left(u_{1}\right), u_{2}\right)+\left(u_{2},-\Lambda u_{1}-V^{\prime}\left(u_{1}\right)\right)=0 .
$$

Finally, using the above definition of $G$ and the fact that the operator $Q$ is self-adjoint, the last term in the above formula is seen to be equal to

$$
\frac{1}{2} \int_{0}^{t} \operatorname{Tr}\left(Q^{1 / 2}\left(Q^{1 / 2}\right)^{*}\right) \mathrm{d} s=t \frac{1}{2} \operatorname{Tr}(Q) .
$$

This shows the trace formula (17) for the exact solution of our problem. 
Remark 6. Similarly to the above computations, for the case of multiplicative noise, one would obtain

$$
\begin{aligned}
H(X(t))= & H(X(0))+\int_{0}^{t}\left(H^{\prime}(X(s)), G(X(s)) \mathrm{d} W(s)\right)+\int_{0}^{t}\left(H^{\prime}(X(s)), A X+F(X)\right) \mathrm{d} s \\
& +\frac{1}{2} \int_{0}^{t} \operatorname{Tr}\left[H^{\prime \prime}(X(s))\left(G(X(s)) Q^{1 / 2}\right)\left(G(X(s)) Q^{1 / 2}\right)^{*}\right] \mathrm{d} s \\
= & H(X(0))+\int_{0}^{t}\left(H^{\prime}(X(s)), G(X(s)) \mathrm{d} W(s)\right) \\
& +\frac{1}{2} \int_{0}^{t} \operatorname{Tr}\left(g\left(u_{1}(s)\right) Q^{1 / 2}\left(g\left(u_{1}(s)\right) Q^{1 / 2}\right)^{*}\right) \mathrm{d} s .
\end{aligned}
$$

Taking expectation thus leads to

$$
\mathbb{E}[H(X(t))]=\mathbb{E}[H(X(0))]+\frac{1}{2} \int_{0}^{t} \mathbb{E}\left[\operatorname{Tr}\left(g\left(u_{1}(s)\right) Q\left(g\left(u_{1}(s)\right)\right)^{*}\right)\right] \mathrm{d} s,
$$

which, for general multiplicative noise, do not give a tractable expression of the drift in the trace formula.

Remark 7. The trace formula is also related to the energy equation, a tool that can be used to analyze the existence, or nonexistence, of solutions to stochastic nonlinear wave equations; see [3] for further details on this topic.

We next observe that, for the finite element solution $X_{h}$, one has

$$
H\left(X_{h}\right)=\frac{1}{2}\left\|u_{h, 2}\right\|^{2}+\frac{1}{2}\left\|\Lambda_{h}^{1 / 2} u_{h, 1}\right\|^{2}+\int_{\mathcal{D}} V\left(u_{h, 1}\right) \mathrm{d} x,
$$

because $\left\|\nabla v_{h}\right\|=\left\|\Lambda^{1 / 2} v_{h}\right\|=\left\|\Lambda_{h}^{1 / 2} v_{h}\right\|$ for finite element functions $v_{h}$. This results from the definitions of $\Lambda^{1 / 2}$ and $\Lambda_{h}^{1 / 2}$; see section 2. Using arguments similar to the proof of Proposition 5, one can now show that the finite element solution $X_{h}(t)$, defined in (7), also possesses a trace formula.

Proposition 8. Let $f, g$, and $W$ be as in Proposition 5. The solution of the finite element approximation of problem $(1), X_{h}(t)$ in $(7)$, satisfies the trace formula

$$
\mathbb{E}\left[H\left(X_{h}(t)\right)\right]=\mathbb{E}\left[H\left(X_{h}(0)\right)\right]+t \frac{1}{2} \operatorname{Tr}\left(\mathcal{P}_{h} Q \mathcal{P}_{h}\right), \quad t \geq 0 .
$$

We will now prove that the full discretization of the stochastic wave equation, that is, the numerical solution given by (16), satisfies an almost trace formula. Indeed, as seen in the theorem below, we get a small defect of size $\mathcal{O}\left(k^{\min (2(\beta-1), 1)}\right)$. However, due to the use of Gronwall's inequality, the defect term is not uniform in time.

Theorem 9. Let $f, g$, and $W$ be as in Propositions 5 and 8. Let further the assumptions in Theorem 4 be fulfilled with $\beta \in[1,2]$. Then the stochastic trigonometric method (16) satisfies an almost trace formula

$$
\mathbb{E}\left[H\left(U^{n}\right)\right]=\mathbb{E}\left[H\left(U^{0}\right)\right]+t_{n} \frac{1}{2} \operatorname{Tr}\left(\mathcal{P}_{h} Q \mathcal{P}_{h}\right)+\mathcal{O}\left(k^{\min (2(\beta-1), 1)}\right)
$$

for $0 \leq t_{n} \leq T$ and $\beta \in[1,2]$. 
Proof. The proof uses techniques similar to those used to prove the mean-square error estimates for the numerical solution in section 3 .

To prove the almost trace formula (19), we first add and subtract the expectation of the Hamiltonian for the finite element solution $X_{h}(t)$

$$
\begin{aligned}
\mathbb{E}\left[H\left(U^{n}\right)\right] & =\mathbb{E}\left[H\left(U^{n}\right)-H\left(X_{h}\left(t_{n}\right)\right)\right]+\mathbb{E}\left[H\left(X_{h}\left(t_{n}\right)\right)\right] \\
& =\mathbb{E}\left[H\left(U^{n}\right)-H\left(X_{h}\left(t_{n}\right)\right)\right]+\mathbb{E}\left[H\left(X_{h}(0)\right)\right]+t_{n} \frac{1}{2} \operatorname{Tr}\left(\mathcal{P}_{h} Q \mathcal{P}_{h}\right)
\end{aligned}
$$

using Proposition 8. We will next show that

$$
\mathbb{E}\left[H\left(U^{n}\right)-H\left(X_{h}\left(t_{n}\right)\right)\right]=\mathcal{O}\left(k^{\min (2(\beta-1), 1)}\right)
$$

for $\beta \in[1,2]$. Indeed, we have that

$$
\begin{aligned}
\mathbb{E}\left[H\left(U^{n}\right)-H\left(X_{h}\left(t_{n}\right)\right)\right]=\mathbb{E}[ & \frac{1}{2} \int_{\mathcal{D}}\left(\left|U_{2}^{n}\right|^{2}-\left|u_{h, 2}\left(t_{n}\right)\right|^{2}\right) \mathrm{d} x \\
& +\frac{1}{2} \int_{\mathcal{D}}\left(\left|\Lambda_{h}^{1 / 2} U_{1}^{n}\right|^{2}-\left|\Lambda_{h}^{1 / 2} u_{h, 1}\left(t_{n}\right)\right|^{2}\right) \mathrm{d} x \\
& \left.+\int_{\mathcal{D}}\left(V\left(U_{1}^{n}\right)-V\left(u_{h, 1}\left(t_{n}\right)\right)\right) \mathrm{d} x\right] .
\end{aligned}
$$

Thus we get three terms to estimate. Using the Cauchy-Schwarz inequality, the first term in the above equation can be estimated by (neglecting the factor $\frac{1}{2}$ for ease of presentation)

$$
\begin{aligned}
\left|\mathbb{E}\left[\left\|U_{2}^{n}\right\|^{2}-\left\|u_{h, 2}\left(t_{n}\right)\right\|^{2}\right]\right| & =\left|\mathbb{E}\left[\left(U_{2}^{n}+u_{h, 2}\left(t_{n}\right), U_{2}^{n}-u_{h, 2}\left(t_{n}\right)\right)\right]\right| \\
& \leq\left(\mathbb{E}\left[\left\|U_{2}^{n}+u_{h, 2}\left(t_{n}\right)\right\|_{h, \beta-1}^{2}\right]\right)^{1 / 2}\left(\mathbb{E}\left[\left\|U_{2}^{n}-u_{h, 2}\left(t_{n}\right)\right\|_{h, 1-\beta}^{2}\right]\right)^{1 / 2} \\
& \leq C\left(\mathbb{E}\left[\left\|\Lambda_{h}^{(1-\beta) / 2}\left(U_{2}^{n}-u_{h, 2}\left(t_{n}\right)\right)\right\|^{2}\right]\right)^{1 / 2},
\end{aligned}
$$

where we have used the discrete norm, the fact that the finite element solution $u_{h, 2}(t)$ is bounded in the mean-square sense (see Proposition 3), and the fact that the numerical solution given by the stochastic trigonometric method is also bounded, i. e.,

$$
\mathbb{E}\left[\left\|U_{1}^{n}\right\|_{h, \beta}^{2}+\left\|U_{2}^{n}\right\|_{h, \beta-1}^{2}\right] \leq C<\infty \quad \text { for } \quad n=0,1, \ldots, N-1
$$

The proof of these estimates is similar to that for the finite element solution given in Proposition 3, except that we now have a sum of integrals of length $k$. This does not cause a problem, since we can simply use the triangle inequality for the deterministic integrals, and for the stochastic integrals we use the property that they are independent with expected value 0 .

Using the definition of the time integrator and techniques similar to the proof of 
the mean-square convergence, one next estimates

$$
\begin{aligned}
\Lambda_{h}^{(1-\beta) / 2}\left(U_{2}^{n}-u_{h, 2}\left(t_{n}\right)\right)= & \sum_{j=0}^{n-1} \int_{t_{j}}^{t_{j+1}} \Lambda_{h}^{(1-\beta) / 2}\left(C_{h}\left(t_{n}-t_{j}\right)-C_{h}\left(t_{n}-s\right)\right) \mathcal{P}_{h} \mathrm{~d} W(s) \\
& +\sum_{j=0}^{n-1} \int_{t_{j}}^{t_{j+1}} \Lambda_{h}^{(1-\beta) / 2} C_{h}\left(t_{n}-t_{j}\right) \mathcal{P}_{h}\left(f\left(U_{1}^{j}\right)-f\left(u_{h, 1}\left(t_{j}\right)\right)\right) \mathrm{d} s \\
& +\sum_{j=0}^{n-1} \int_{t_{j}}^{t_{j+1}} \Lambda_{h}^{(1-\beta) / 2} C_{h}\left(t_{n}-t_{j}\right) \mathcal{P}_{h}\left(f\left(u_{h, 1}\left(t_{j}\right)\right)-f\left(u_{h, 1}(s)\right)\right) \mathrm{d} s \\
& +\sum_{j=0}^{n-1} \int_{t_{j}}^{t_{j+1}} \Lambda_{h}^{(1-\beta) / 2}\left(C_{h}\left(t_{n}-t_{j}\right)-C_{h}\left(t_{n}-s\right)\right) \mathcal{P}_{h} f\left(u_{h, 1}(s)\right) \mathrm{d} s \\
= & : J_{1}+J_{2}+J_{3}+J_{4} .
\end{aligned}
$$

Using the temporal regularity of the cosine operator (see (12)), equation (14), and assumptions (9) (recall that $g=1$ here), one gets

$$
\begin{aligned}
\mathbb{E}\left[\left\|J_{1}\right\|^{2}\right]= & \sum_{j=0}^{n-1} \int_{t_{j}}^{t_{j+1}}\left\|\Lambda_{h}^{(1-\beta)}\left(C_{h}\left(t_{n}-t_{j}\right)-C_{h}\left(t_{n}-s\right)\right) \Lambda_{h}^{(\beta-1) / 2} \mathcal{P}_{h}\right\|_{\mathcal{L}_{2}^{0}}^{2} \mathrm{~d} s \\
\leq & C \sum_{j=0}^{n-1} \int_{t_{j}}^{t_{j+1}} \| \Lambda_{h}^{(1-\beta)}\left(C_{h}\left(t_{n}-t_{j}\right)-C_{h}\left(t_{n}-s\right)\right) \Lambda_{h}^{(\beta-1) / 2} \mathcal{P}_{h} \Lambda^{-(\beta-1) / 2} \\
& \quad \times \Lambda^{(\beta-1) / 2} Q^{1 / 2} \|_{\mathrm{HS}}^{2} \mathrm{~d} s \\
\leq & C k^{2 \min (2(\beta-1), 1)} \quad \text { for } \quad \beta \in[1,2] .
\end{aligned}
$$

Next, using the convergence results from Theorem 4 and the Lipschitz assumption on $f$, we observe that

$$
\begin{aligned}
\left(\mathbb{E}\left[\left\|J_{2}\right\|^{2}\right]\right)^{1 / 2} & \leq \sum_{j=0}^{n-1} \int_{t_{j}}^{t_{j+1}}\left(\mathbb{E}\left[\left\|\Lambda_{h}^{(1-\beta) / 2} C_{h}\left(t_{n}-t_{j}\right) \mathcal{P}_{h}\left(f\left(U_{1}^{j}\right)-f\left(u_{h, 1}\left(t_{j}\right)\right)\right)\right\|^{2}\right]\right)^{1 / 2} \mathrm{~d} s \\
& \leq C k \sum_{j=0}^{n-1}\left(\mathbb{E}\left[\left\|U_{1}^{j}-u_{h, 1}\left(t_{j}\right)\right\|^{2}\right]\right)^{1 / 2} \leq C k^{\min (\beta, 1)} \quad \text { for } \quad \beta \in[1,2] .
\end{aligned}
$$

Similarly, using the assumptions on $f$ given in (9) and the regularity property of the finite element solution stated in Proposition 3, one gets

$$
\begin{aligned}
\left(\mathbb{E}\left[\left\|J_{3}\right\|^{2}\right]\right)^{1 / 2} & \leq \sum_{j=0}^{n-1} \int_{t_{j}}^{t_{j+1}}\left(\mathbb{E}\left[\left\|\Lambda_{h}^{(1-\beta) / 2} C_{h}\left(t_{n}-t_{j}\right) \mathcal{P}_{h}\left(f\left(u_{h, 1}\left(t_{j}\right)\right)-f\left(u_{h, 1}(s)\right)\right)\right\|^{2}\right]\right)^{1 / 2} \mathrm{~d} s \\
& \leq C k^{\min (\beta, 1)} \quad \text { for } \quad \beta \in[1,2] .
\end{aligned}
$$

For the last term, $J_{4}$, we obtain the estimate for $\beta \in[1,2]$ as follows:

$$
\begin{gathered}
\left(\mathbb{E}\left[\left\|J_{4}\right\|^{2}\right]\right)^{1 / 2} \leq \sum_{j=0}^{n-1} \int_{t_{j}}^{t_{j+1}}\left(\mathbb { E } \left[\| \Lambda_{h}^{(1-\beta)}\left(C_{h}\left(t_{n}-t_{j}\right)-C_{h}\left(t_{n}-s\right)\right)\right.\right. \\
\left.\left.\quad \times \Lambda_{h}^{(\beta-1) / 2} \mathcal{P}_{h} f\left(u_{h, 1}(s)\right) \|^{2}\right]\right)^{1 / 2} \mathrm{~d} s \\
\leq C k^{\min (2(\beta-1), 1)},
\end{gathered}
$$

Copyright $\odot$ by SIAM. Unauthorized reproduction of this article is prohibited. 
where we have used (12), the equivalence between the norms (15), the assumptions on the nonlinearity $f$, and the fact that the finite element solution $u_{h, 1}$ is bounded in the norm $\|\cdot\|_{h, \beta-1}$.

Collecting all of the above estimates and observing that $2(\beta-1) \leq \beta$ for $\beta \in[1,2]$, we finally get

$$
\left|\mathbb{E}\left[\left\|U_{2}^{n}\right\|^{2}-\left\|u_{h, 2}\left(t_{n}\right)\right\|^{2}\right]\right| \leq C k^{\min (2(\beta-1), 1)} \quad \text { for } \quad \beta \in[1,2] .
$$

The second term in (21) can be estimated in a similar way. We have

$$
\begin{aligned}
\left|\mathbb{E}\left[\left\|\Lambda_{h}^{1 / 2} U_{1}^{n}\right\|^{2}-\left\|\Lambda_{h}^{1 / 2} u_{h, 1}\left(t_{n}\right)\right\|^{2}\right]\right| & \leq\left(\mathbb{E}\left[\left\|U_{1}^{n}+u_{h, 1}\left(t_{n}\right)\right\|_{h, \beta}^{2}\right]\right)^{1 / 2}\left(\mathbb{E}\left[\left\|U_{1}^{n}-u_{h, 1}\left(t_{n}\right)\right\|_{h, 2-\beta}^{2}\right]\right)^{1 / 2} \\
& \leq C\left(\mathbb{E}\left[\left\|\Lambda_{h}^{(2-\beta) / 2}\left(U_{1}^{n}-u_{1, h}\left(t_{n}\right)\right)\right\|^{2}\right]\right)^{1 / 2} \\
& \leq C k^{\min (2(\beta-1), 1)} \quad \text { for } \beta \in[1,2] .
\end{aligned}
$$

When estimating $\mathbb{E}\left[\left\|\Lambda_{h}^{(2-\beta) / 2}\left(U_{1}^{n}-u_{1, h}\left(t_{n}\right)\right)\right\|^{2}\right]$ we get the same terms as $J_{1}$ through $J_{4}$ above, except that cosine is replaced by sine everywhere. Hence, the same estimate holds. For the third and final term in (21), using the mean value theorem we get

$$
\begin{aligned}
\mathbb{E}\left[\left\|V\left(U_{1}^{n}\right)-V\left(u_{h, 1}\left(t_{n}\right)\right)\right\|_{L_{1}(\mathcal{D})}\right] & \leq C \mathbb{E}\left[\left\|V\left(U_{1}^{n}\right)-V\left(u_{h, 1}\left(t_{n}\right)\right)\right\|_{L_{2}(\mathcal{D})}\right] \\
& \leq C\left\|V^{\prime}(\xi)\left(U_{1}^{n}-u_{h, 1}\left(t_{n}\right)\right)\right\|_{L_{2}\left(\Omega, \dot{H}^{0}\right)}
\end{aligned}
$$

Recalling that $f(u)=-V^{\prime}(u)$ and using Hölder's inequality, the fact that the numerical solutions are bounded in the mean-square sense, and the error bounds stated in Theorem 4, we next estimate the following expression:

$$
\begin{aligned}
\mathbb{E}\left[\left\|V\left(U_{1}^{n}\right)-V\left(u_{h, 1}\left(t_{n}\right)\right)\right\|_{L_{1}(\mathcal{D})}\right] & \leq C\left\|V^{\prime}(\xi)\left(U_{1}^{n}-u_{h, 1}\left(t_{n}\right)\right)\right\|_{L_{2}\left(\Omega, \dot{H}^{0}\right)} \\
& \leq C\left(\mathbb{E}\left[\left\|U_{1}^{n}-u_{h, 1}\left(t_{n}\right)\right\|_{L_{2}(\mathcal{D})}^{2}\right]\right)^{1 / 2} \leq C k^{\min (\beta, 1)} .
\end{aligned}
$$

Putting all of these estimates together, we obtain (20), and the theorem is proved.

5. Numerical experiments. This section illustrates numerically the main results of the paper. We first present the time integrators we will consider, then test their mean-square orders of convergence on various problems, and finally illustrate their behaviors with respect to the trace formula from the previous section.

5.1. Setting. The solution of our stochastic wave equation (1) will now be numerically approximated using the method of lines, i.e., with a linear finite element method in space and then with various time integrators (see below). Further, we will consider two kinds of noise: space-time white noise with covariance operator $Q=I$ and correlated noise with $Q=\Lambda^{-s}$ for some $s>0$. We refer the reader to [5] for a discussion on the approximation of the noise.

We shall compare the stochastic trigonometric method (16) with the following classical numerical schemes for stochastic differential equations. When applied to the wave equation in the form (2), these numerical integrators are as follows:

1. The forward Euler-Maruyama scheme (see, for example, [14] or [18]),

$$
X^{n+1}=X^{n}+k A X^{n}+k F\left(X^{n}\right)+G\left(X^{n}\right) \Delta W^{n} .
$$

2. The semi-implicit Euler-Maruyama scheme (see, for example, [11] or [20]),

$$
X^{n+1}=X^{n}+k A X^{n+1}+k F\left(X^{n}\right)+G\left(X^{n}\right) \Delta W^{n} .
$$

Copyright (c) by SIAM. Unauthorized reproduction of this article is prohibited. 
3. The backward Euler-Maruyama scheme (see, for example, [14] or [18]),

$$
X^{n+1}=X^{n}+k A X^{n+1}+k F\left(X^{n+1}\right)+G\left(X^{n}\right) \Delta W^{n} .
$$

4. The semi-implicit Crank-Nicolson-Maruyama scheme (see [11] or [20]),

$$
X^{n+1}=X^{n}+\frac{k}{2} A\left(X^{n+1}+X^{n}\right)+k F\left(X^{n}\right)+G\left(X^{n}\right) \Delta W^{n} .
$$

Note that the backward Euler-Maruyama scheme, the semi-implicit Euler-Maruyama scheme, and the semi-implicit Crank-Nicolson-Maruyama scheme are implicit numerical integrators.

All of the numerical experiments were performed in MATLAB using specially designed software, and the random numbers were generated with the command randn.

5.2. Multiplicative noise. Let us first consider the one-dimensional hyperbolic Anderson model [7, 10],

$$
\begin{array}{ll}
\mathrm{d} \dot{u}(x, t)-u_{x x}(x, t) \mathrm{d} t=u(x, t) \mathrm{d} W(x, t) & \text { for }(x, t) \in(0,1) \times(0,1), \\
u(0, t)=u(1, t)=0, & t \in(0,1), \\
u(x, 0)=\sin (2 \pi x), \dot{u}(x, 0)=\sin (3 \pi x), & x \in(0,1) .
\end{array}
$$

This stochastic partial differential equation with multiplicative noise is now discretized in space by a linear finite element method with mesh size $h$. This leads to a system of stiff stochastic differential equations. The latter problem is then discretized in time by various integrators with time step $k$.

Figure 1 illustrates the results on the spatial discretization of the finite element method as stated in Theorem 4 . The spatial mean-square errors at time $T_{\text {end }}=1$,

$$
\sqrt{\mathbb{E}\left[\left\|u_{h}\left(x, T_{\text {end }}\right)-u\left(x, T_{\text {end }}\right)\right\|^{2}\right]}
$$

are displayed for various values of the parameter $h=2^{-\ell}, \ell=2, \ldots, 9$. The covariance operator is chosen as $Q=\Lambda^{-s}$ for $s=0,1 / 2,1 / 3,1 / 4$. In the present situation, $f(u)=0$ and $g(u)=u$ satisfy assumptions (9) with $\beta<s+\frac{1}{2}$. This can be seen using the computations done in subsection 4.1 from [13] (with $\rho=2 s$ and $\alpha=\frac{\beta-1}{2}$ ). A clear dependence of the spatial convergence rates with respect to the covariance operator can be observed in this figure, in agreement with Theorem 4. Here we simulate the exact solution $u(x, t)$ with the numerical one using the stochastic trigonometric method (STM) (16) with a small time step $k_{\text {exact }}=2^{-9}$ (in order to neglect the error from the discretization in time) and $h_{\text {exact }}=2^{-9}$ for the mesh of the finite element method. The expected values are approximated by computing averages over $M_{\mathrm{s}}=2500$ samples. We computed the estimate for the largest standard errors of all schemes to be 0.0026 . This shows that the error due to a Monte-Carlo approximation is negligible.

We are now interested in the time discretization of the above stochastic partial differential equation with space-time white noise $(Q=I$ and thus $\beta<1 / 2)$. We compute the temporal errors at time $T_{\text {end }}=0.5$. In Figure 2 , one can observe the rates of mean-square convergence of various time integrators. The expected rate of convergence $\mathcal{O}\left(k^{1 / 2}\right)$ of the stochastic trigonometric method as stated in Theorem 4 can be confirmed. Again, the exact solution is approximated by the stochastic trigonometric method with a very small time step $k_{\text {exact }}=2^{-11}$ and uses $h_{\text {exact }}=2^{-9}$ for the spatial 


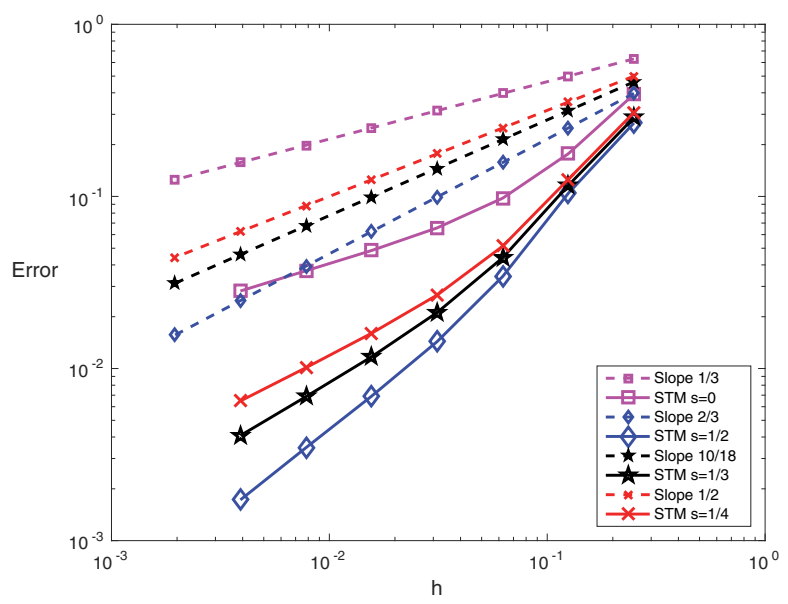

FIG. 1. The Anderson model: Spatial rates of convergence for the covariance operators $Q=$ $\Lambda^{-s}$ with $s=0,1 / 2,1 / 3,1 / 4$. The dotted lines are reference lines of slopes $1 / 3,2 / 3,10 / 18,1 / 2$. $M_{\mathrm{S}}=2500$ samples.

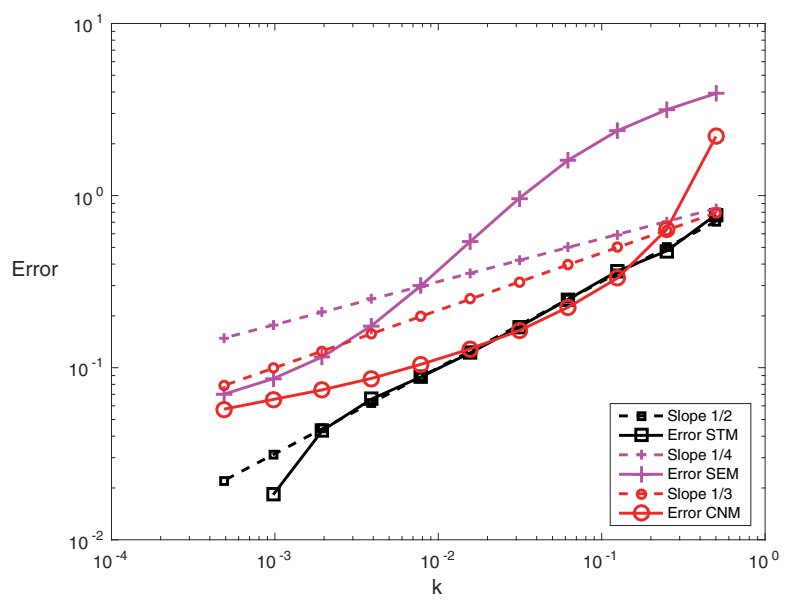

Fig. 2. The Anderson model (space-time white noise): Temporal rates of convergence of the stochastic trigonometric method (STM), the semi-implicit Euler-Maruyama scheme (SEM), and the Crank-Nicolson-Maruyama scheme (CNM). The reference lines have slopes 1/4,1/3, and 1/2. $M_{\mathrm{S}}=2500$ samples.

discretization. $M_{\mathrm{s}}=2500$ samples are used for the approximation of the expected values. We computed the estimate for the largest standard errors of all schemes to be 0.01. The numerical results for the forward and backward Euler-Maruyama schemes are not displayed, since these numerical schemes would have to use very small time steps for such an $h_{\text {exact }}$ (see also subsection 5.5).

5.3. Semilinear problem with additive space-time white noise. We next consider the sine-Gordon equation driven by additive space-time white noise $(Q=I$

Copyright $\odot$ by SIAM. Unauthorized reproduction of this article is prohibited. 


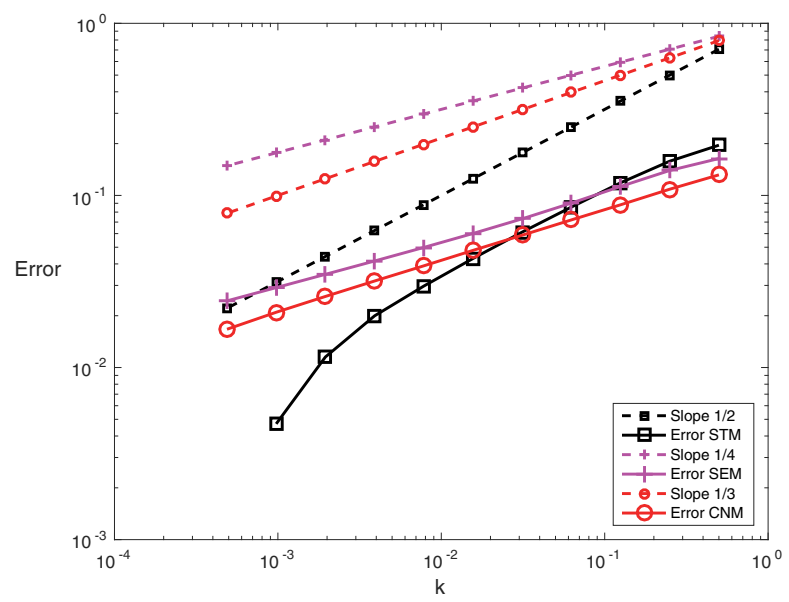

FIG. 3. The sine-Gordon equation (space-time white noise): Temporal rates of convergence of the stochastic trigonometric method (STM), the semi-implicit Euler-Maruyama scheme (SEM), and the Crank-Nicolson-Maruyama scheme (CNM). The dotted lines have slopes 1/4,1/3, and 1/2. $M_{\mathrm{S}}=2500$ samples.

and thus $\beta<1 / 2)$

$$
\begin{array}{ll}
\mathrm{d} \dot{u}(x, t)-u_{x x}(x, t) \mathrm{d} t=-\sin (u(x, t)) \mathrm{d} t+\mathrm{d} W(x, t), & (x, t) \in(0,1) \times(0,0.5), \\
u(0, t)=u(1, t)=0, & t \in(0,0.5), \\
u(x, 0)=0, \dot{u}(x, 0)=1_{\left[\frac{1}{4}, \frac{3}{4}\right]}(x), & x \in(0,1),
\end{array}
$$

where $1_{I}(x)$ denotes the indicator function for the interval $I$.

Figure 3 displays the rates of mean-square convergence at $T_{\mathrm{end}}=0.5$ of various time integrators. The expected temporal rate of convergence $\mathcal{O}\left(k^{1 / 2}\right)$ of the stochastic trigonometric method as stated in Theorem 4 can be confirmed. Again, the exact solution is approximated by the stochastic trigonometric method with a very small step size $k_{\text {exact }}=2^{-11}$ and uses $h_{\text {exact }}=2^{-9}$ for the spatial discretization. $M_{\mathrm{s}}=2500$ samples are used for the approximation of the expected values. We computed the estimate for the largest standard errors for all schemes to be 0.0027 , showing that the error due to a Monte-Carlo approximation is negligible.

5.4. Semilinear equation with multiplicative noise. In this subsection, we consider the sine-Gordon equation driven by multiplicative space-time white noise $(Q=I$ and thus $\beta<1 / 2)$

$$
\begin{array}{ll}
\mathrm{d} \dot{u}(x, t)-u_{x x}(x, t) \mathrm{d} t=-\sin (u(x, t)) \mathrm{d} t+u(x, t) \mathrm{d} W(x, t), & (x, t) \in(0,1) \times(0,0.5), \\
u(0, t)=u(1, t)=0, & t \in(0,0.5), \\
u(x, 0)=\sin (2 \pi x), \dot{u}(x, 0)=\sin (3 \pi x), & x \in(0,1) .
\end{array}
$$

Figure 4 displays the rates of mean-square convergence of various time integrators when applied to this semilinear problem with multiplicative noise. The expected temporal rate of convergence $\mathcal{O}\left(k^{1 / 2}\right)$ of the stochastic trigonometric method as stated in Theorem 4 can be confirmed. One also observes a slower convergence rate for the other integrators. As before, a reference solution is computed by the stochastic trigonometric method with a very small step size $k_{\text {exact }}=2^{-11}$ and uses $h_{\text {exact }}=2^{-9}$ 


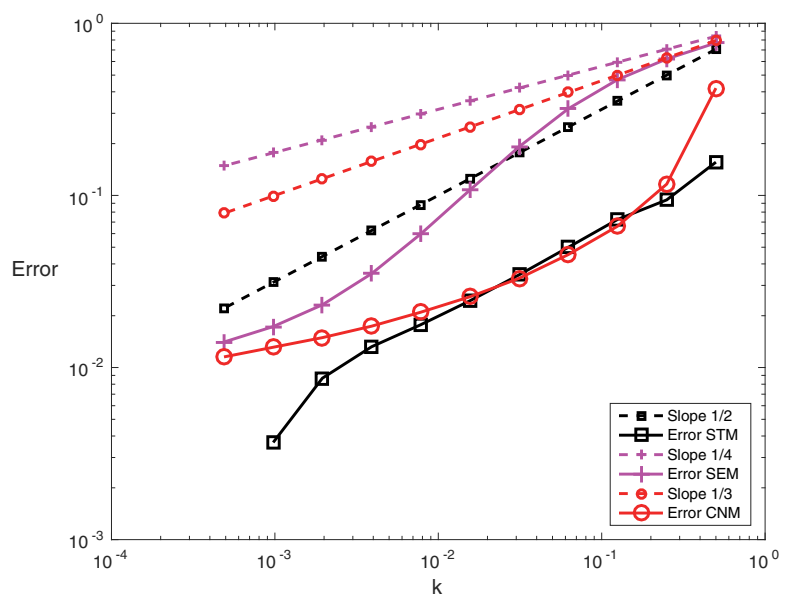

FIG. 4. The sine-Gordon equation with multiplicative space-time white noise: Temporal rates of convergence of the stochastic trigonometric method (STM), the semi-implicit Euler-Maruyama scheme (SEM), and the Crank-Nicolson-Maruyama scheme (CNM). The dotted lines have slopes $1 / 4,1 / 3$, and $1 / 2 . M_{\mathrm{s}}=2500$ samples.

for the spatial discretization. $M_{\mathrm{s}}=2500$ samples are used for the approximation of the expected values. We computed the estimate for the largest standard errors of all schemes to be 0.006 . This shows that the error due to a Monte-Carlo approximation is negligible.

5.5. Trace formula. We will now illustrate the trace formula from section 4 . To do this, we again consider the above sine-Gordon equation with additive noise and solve this problem with a linear finite element method in space, and in time we use the stochastic trigonometric method (16) with $f(u)=-\sin (u), g(u)=1$. Figure 5 (top) displays the expected value of the Hamiltonian along the numerical solutions of the above stochastic sine-Gordon equation, where the covariance operator is given by $Q=\Lambda^{-2}$. In the present situation, the Lipschitz function $f(u)=-\sin (u)$ and the function $g(u)=1$ satisfy assumptions (9) with $\beta=2$. This is seen using the fact that the eigenvalues of the Laplace operator with Dirichlet boundary conditions satisfy $\lambda_{j} \sim j^{2}$, and the eigenvectors are given by $\{\sqrt{2} \sin (j \pi x)\}_{j}$. The meshes are $h=0.1$ and $k=0.01$, the time interval is $[0,5]$, and $M_{\mathrm{s}}=2500$ samples are used for the approximation of the expected values. For this experiment, the largest standard errors for all of the numerical schemes (except for the Euler-Maruyama scheme) is of size 0.002, confirming that the Monte-Carlo errors are negligible. In this figure, one can observe the unsatisfactory behavior of classical Euler-Maruyama-type methods. This is not a big surprise, since, already for stochastic ordinary differential equations, the growth rate of the expected energy along solutions given by these numerical solutions is incorrect $[19,4]$. The Crank-Nicolson-Maruyama scheme, however, seems to reproduce very well the linear drift in the expected value of the Hamiltonian. Let us see what happens when one uses a bigger time step and a longer time interval. Figure 5 (bottom) displays the expected energies on the longer time interval $[0,250]$ for the Crank-Nicolson-Maruyama scheme and the stochastic trigonometric method with a larger time step $k=0.1$. The other parameters are the same as in the above numerical experiment; in particular, the Monte-Carlo error for the stochastic trigonometric method is negligible (error of size 0.01). On this long-time interval, excellent 

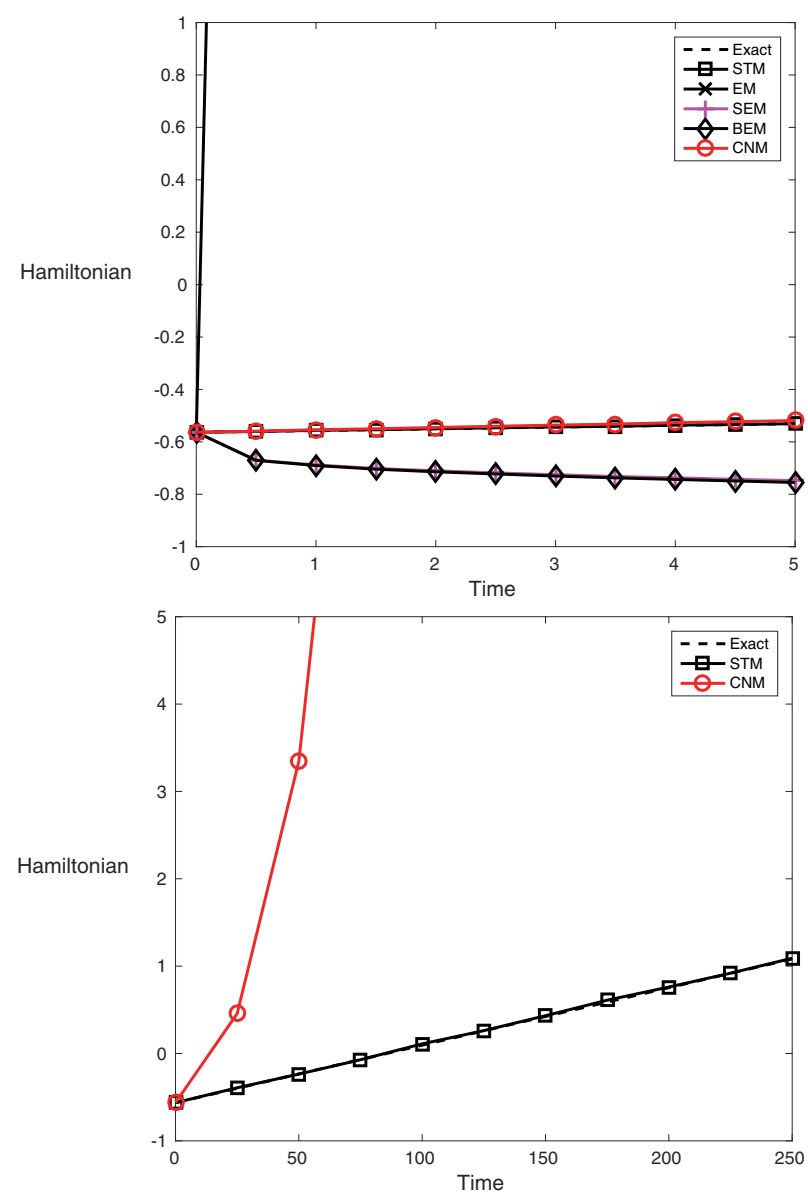

FIG. 5. Trace formula for the sine-Gordon equation: Expected values of the Hamiltonian along the numerical solutions given by the stochastic trigonometric method (STM), the forward EulerMaruyama scheme (EM), the semi-implicit Euler-Maruyama scheme (SEM), the backward EulerMaruyama scheme (BEM), and the Crank-Nicolson-Maruyama scheme (CNM). $M_{\mathrm{S}}=2500$ samples. Mesh size: $h=0.1$. Time intervals and time steps: $[0,5], k=0.01$ (top) and $[0,250], k=0.1$ (bottom).

behavior of the stochastic trigonometric method (16) is still observed, although this does not follow from the result presented in Theorem 9.

6. Appendix. In order to improve the readability of the paper, we give some details for the proofs of the results in section 2. The proofs of (10) and (11) can be found in Corollary 4.2 in [16]. They are obtained by interpolation between the results for the endpoints of the parameter values. These in turn are well-known estimates for the finite element approximation of the homogeneous wave equation: $\ddot{u}_{h}+\Lambda_{h} u_{h}=0, t \geq 0 ; u_{h}(0)=\mathcal{R}_{h} u_{0}, \dot{u}_{h}(0)=\mathcal{P}_{h} v_{0}$.

For example, for $\gamma=1$ we have, by a standard stability estimate,

$$
\begin{aligned}
\left\|\mathcal{G}_{h}(t) X_{0}\right\| & \leq C\left(\left\|\mathcal{R}_{h} u_{0}\right\|+\left\|\mathcal{P}_{h} v_{0}\right\|_{-1, h}+\left\|u_{0}\right\|+\left\|v_{0}\right\|_{-1}\right) \\
& \leq C\left(\left\|u_{0}\right\|_{1}+\left\|v_{0}\right\|_{-1}\right) \leq C h^{0}\left\|X_{0}\right\| \|_{1}
\end{aligned}
$$

Copyright (c) by SIAM. Unauthorized reproduction of this article is prohibited. 
since $\mathcal{R}_{h}$ is not bounded with respect to the $\dot{H}^{0}$-norm. For $\gamma=3$, we have

$$
\left\|\mathcal{G}_{h}(t) X_{0}\right\| \leq C(1+t) h^{2}\left(\left\|u_{0}\right\|_{3}+\left\|v_{0}\right\|_{2}\right) \leq C(1+t) h^{2}\left\|X_{0}\right\|_{3} ;
$$

cf. the estimation of $F_{h}$ in the proof of Corollary 4.2 in [16]. Interpolation between these two cases completes the proof of the first bound in (10). Note that the required initial regularity is one order higher than the order of convergence. This is typical of the finite element method for the wave equation. Another choice of projector, $u_{h}(0)=\mathcal{P}_{h} u_{0}$, would give a slightly better result for low initial regularity here, but a worse result for $\dot{\mathcal{G}}_{h}$.

Acknowledgment. We appreciate the referees' comments on an earlier version of the paper.

\section{REFERENCES}

[1] A. Andersson and S. Larsson, Weak convergence for a spatial approximation of the nonlinear stochastic heat equation, Math. Comp., 85 (2016), pp. 1335-1358. doi:10.1090/mcom/3016.

[2] B. P. Belinskiy and P. Caithamer, Energy of an elastic mechanical system driven by Gaussian noise white in time, in Dynamical Systems and Differential Equations (Kennesaw, GA, 2000), Discrete Contin. Dynam. Systems, 2001, Added Volume, pp. 39-49.

[3] P.-L. Chow, Stochastic wave equations with polynomial nonlinearity, Ann. Appl. Probab., 12 (2002), pp. 361-381. doi:10.1214/aoap/1015961168.

[4] D. Cohen, On the numerical discretisation of stochastic oscillators, Math. Comput. Simul., 82 (2012), pp. 1478-1495. doi:10.1016/j.matcom.2012.02.004.

[5] D. Cohen, S. Larsson, And M. Sigg, A trigonometric method for the linear stochastic wave equation, SIAM J. Numer. Anal., 51 (2013), pp. 204-222. doi:10.1137/12087030X.

[6] D. Cohen And L. Quer-Sardanyons, A fully discrete approximation of the onedimensional stochastic wave equation, IMA J. Numer. Anal., 36 (2016), pp. 400-420. doi:10.1093/imanum/drv006.

[7] D. Conus And R. C. Dalang, The non-linear stochastic wave equation in high dimensions, Electron. J. Probab., 13 (2008), pp. 629-670. doi:10.1214/EJP.v13-500.

[8] G. Da Prato And J. ZabczYK, Stochastic Equations in Infinite Dimensions, Encyclopedia Math. Appl. 44, Cambridge University Press, Cambridge, UK, 1992. doi:10.1017/CBO9780511666223.

[9] R. C. Dalang, The stochastic wave equation, in A Minicourse on Stochastic Partial Differential Equations, Lecture Notes in Math. 1962, Springer-Verlag, Berlin, 2009, pp. 39-71. doi:10.1007/978-3-540-85994-9_2.

[10] R. C. DAlang AND C. Mueller, Intermittency properties in a hyperbolic Anderson problem, Ann. Inst. Henri Poincaré Probab. Stat., 45 (2009), pp. 1150-1164. doi:10.1214/08-AIHP199.

[11] E. Hausenblas, Approximation for semilinear stochastic evolution equations, Potential Anal., 18 (2003), pp. 141-186. doi:10.1023/A:1020552804087.

[12] A. JentZen AND P. E. Kloeden, Overcoming the order barrier in the numerical approximation of stochastic partial differential equations with additive space-time noise, Proc. R. Soc. Lond. Ser. A Math. Phys. Eng. Sci., 465 (2009), pp. 649-667. doi:10.1098/rspa.2008.0325.

[13] A. Jentzen And M. RöCKNeR, Regularity analysis for stochastic partial differential equations with nonlinear multiplicative trace class noise, J. Differential Equations, 252 (2012), pp. 114-136. doi:10.1016/j.jde.2011.08.050.

[14] P. E. Kloeden and E. Platen, Numerical Solution of Stochastic Differential Equations, Appl. Math. (New York) 23, Springer-Verlag, Berlin, 1992.

[15] M. Kovács, S. Larsson, And F. Lindgren, Weak convergence of finite element approximations of linear stochastic evolution equations with additive noise, BIT, 52 (2012), pp. 85108.

[16] M. Kovács, S. Larsson, And F. Saedpanah, Finite element approximation of the linear stochastic wave equation with additive noise, SIAM J. Numer. Anal., 48 (2010), pp. 408427. doi:10.1137/090772241.

[17] G. J. Lord ANd A. TAmbue, Stochastic exponential integrators for the finite element discretization of SPDEs for multiplicative and additive noise, IMA J. Numer. Anal., 33 (2013), pp. 515-543. doi:10.1093/imanum/drr059.

Copyright (c) by SIAM. Unauthorized reproduction of this article is prohibited. 
[18] G. N. Milstein and M. V. Tretyakov, Stochastic Numerics for Mathematical Physics, Sci. Comput., Springer-Verlag, Berlin, 2004.

[19] A. H. Strømmen Melb $\varnothing$ and D. J. Higham, Numerical simulation of a linear stochastic oscillator with additive noise, Appl. Numer. Math., 51 (2004), pp. 89-99.

[20] J. B. WALSH, Finite element methods for parabolic stochastic PDE's, Potential Anal., 23 (2005), pp. 1-43. doi:10.1007/s11118-004-2950-y.

[21] X. WAng, An exponential integrator scheme for time discretization of nonlinear stochastic wave equation, J. Sci. Comput., 64 (2015), pp. 234-263. doi:10.1007/s10915-014-9931-0.

[22] X. WANG, S. GAN, AND J. TANG, Higher order strong approximations of semilinear stochastic wave equation with additive space-time white noise, SIAM J. Sci. Comput., 36 (2014), pp. A2611-A2632. doi:10.1137/130937524.

[23] Y. YAN, Galerkin finite element methods for stochastic parabolic partial differential equations, SIAM J. Numer. Anal., 43 (2005), pp. 1363-1384. doi:10.1137/040605278.

Copyright (c) by SIAM. Unauthorized reproduction of this article is prohibited. 